\title{
Rotating black hole entropy from M5-branes
}

\author{
Francesco Benini, ${ }^{a, b, c}$ Dongmin Gang ${ }^{d, e}$ and Leopoldo A. Pando Zayas ${ }^{c, f}$ \\ ${ }^{a}$ SISSA, \\ Via Bonomea 265, 34136 Trieste, Italy \\ ${ }^{b}$ INFN, Sezione di Trieste, \\ Via Valerio 2, 34127 Trieste, Italy \\ ${ }^{c} I C T P$, \\ Strada Costiera 11, 34151 Trieste, Italy \\ ${ }^{d}$ Quantum Universe Center, Korea Institute for Advanced Study, \\ Seoul 02455, Korea \\ ${ }^{e}$ Asia Pacific Center for Theoretical Physics (APCTP), \\ Headquarters San 31, Hyoja-dong, Nam-gu, Pohang 790-784, Korea \\ ${ }^{f}$ Leinweber Center for Theoretical Physics, Randall Laboratory of Physics, \\ The University of Michigan, \\ Ann Arbor, MI 48109-1040, U.S.A. \\ E-mail: fbenini@sissa.it, dongmin.gang@apctp.org, lpandoz@umich.edu
}

AbStRaCt: We compute the superconformal index of $3 \mathrm{~d} \mathcal{N}=2$ superconformal field theories obtained from $N$ M5-branes wrapped on a hyperbolic 3-manifold. Exploiting the $3 \mathrm{~d}$-3d correspondence, we use perturbative invariants of $\operatorname{SL}(N, \mathbb{C})$ Chern-Simons theory to determine the superconformal index in the large $N$ limit, including corrections logarithmic in $N$. The leading order partition function provides a microscopic foundation for the entropy function of the dual rotating asymptotically $\mathrm{AdS}_{4}$ black holes. We also verify that the supergravity one-loop contribution to the $\log N$ term coincides with the field theoretic result. We propose a $3 \mathrm{~d}-3 \mathrm{~d}$ formulation for the refined topologically twisted index, and provide strong evidence in support of its vanishing - which agrees with the fact that the expected dual rotating magnetically-charged black hole does not exist. This provides an interesting link between gravity and a tantalizing mathematical result.

Keywords: Black Holes in String Theory, Chern-Simons Theories, Gauge-gravity correspondence, Models of Quantum Gravity

ARXIV EPRINT: 1909.11612 


\section{Contents}

1 Introduction 1

$2 \quad 3 d-3 d$ relations for $3 d$ indices $\quad 3$

$2.13 \mathrm{~d} \mathcal{T}_{N}\left[M_{3}\right]$ theory 3

$2.2 \operatorname{SL}(N, \mathbb{C})$ Chern-Simons theory on $M_{3}$

2.3 3d-3d relations 6

$\begin{array}{lll}2.3 .1 & \text { Superconformal index } & 7\end{array}$

2.3.2 Refined twisted index 8

2.3.3 Twisted indices 9

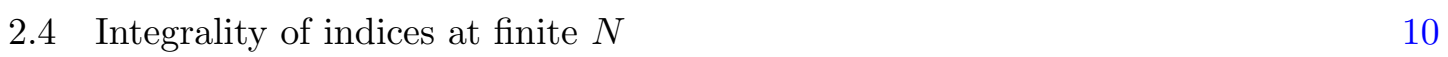

$\begin{array}{lll}2.4 .1 & \text { Vanishing of the refined twisted index } & 10\end{array}$

$\begin{array}{ll}\text { 2.4.2 Integer expansion of the twisted index } & 14\end{array}$

$\begin{array}{lll}3 & \text { Large } N \text { limit of the indices } & 17\end{array}$

$\begin{array}{lll}3.1 & \text { Perturbative Chern-Simons invariants } & 17\end{array}$

$\begin{array}{ll}3.2 \text { Superconformal index } & 19\end{array}$

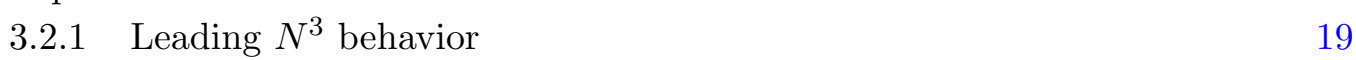

\begin{tabular}{ll}
3.2 .2 & Logarithmic subleading corrections \\
\hline
\end{tabular}

$\begin{array}{lll}3.3 & \text { Twisted indices for } g>1 & 20\end{array}$

$\begin{array}{ll}\text { 3.3.1 All orders in } 1 / N & 21\end{array}$

3.4 Refined index: no $N^{3}$ behavior $\quad 21$

4 M5-branes wrapped on hyperbolic $M_{3}$ and $\mathcal{N}=2$ gauged supergravity 22

4.1 Rotating electrically-charged $\mathrm{AdS}_{4}$ black hole 24

4.2 Rotating magnetically-charged $\mathrm{AdS}_{4}$ black holes 26

5 Logarithmic corrections from one-loop supergravity 26

6 Conclusions 28

$\begin{array}{lll}6.1 & \text { Future directions } & 29\end{array}$

A Perturbative invariants $S_{n}^{\alpha}\left[M_{3} ; N=2\right]$ for some $M_{3}$ 's 30

\section{Introduction}

One of the most basic quantum properties of black holes is that they have an entropy [1-5]. Bekenstein and Hawking understood that at leading order, the entropy in Einstein gravity is fixed in terms of the horizon area by the simple formula $S_{\mathrm{BH}}=$ Area $/ 4 G_{\mathrm{N}}$ where $G_{\mathrm{N}}$ is the Newton constant. This formula is universal: it applies in all dimensions, and for all 
types of smooth black holes. One of the great successes of string theory was to reproduce the Bekenstein-Hawking entropy of certain asymptotically-flat BPS black holes through a microscopic computation [6].

In the context of asymptotically $\mathrm{AdS}_{4}$ black holes, string theory in its AdS/CFT guise has again provided a microscopic explanation for the Bekenstein-Hawking entropy [7, 8]. Namely, it has been shown that the topologically twisted index [9] of a certain field theory precisely matches the entropy function (i.e., the grand canonical partition function) of the dual magnetically-charged asymptotically $\mathrm{AdS}_{4}$ black holes. Similar microscopic explanations have now been extended to a wide range of contexts, e.g., in four [10-14], five [15-18], six [19-22] and seven [23] dimensions (see the review [24] for a more complete list of references).

For rotating electrically-charged asymptotically $\mathrm{AdS}_{4}$ black holes an impressive result was reported based on field theoretic computations of the superconformal index [25]; more recently an explanation based on localization was provided in [26]. In this manuscript our goal is to approach a class of rotating, electrically-charged asymptotically $\mathrm{AdS}_{4}$ black holes via their dual field theory. We concentrate on configurations that are related via dual descriptions to a stack of $N$ M5-branes. An advantage of this approach is that it allows us to make use of the $3 \mathrm{~d}-3 \mathrm{~d}$ correspondence $[27,28]$. Recall that the $3 \mathrm{~d}-3 \mathrm{~d}$ correspondence states an equivalence between certain $3 \mathrm{~d} \mathcal{N}=2$ superconformal field theories $\mathcal{T}_{N}\left[M_{3}\right]$ and Chern-Simons theory with gauge group $\operatorname{SL}(N, \mathbb{C})$ on hyperbolic three-manifolds $M_{3}$. This correspondence has the potential to provide an expression, exact in $N$, for various partition functions of the field theories through known Chern-Simons results. Most of the field theoretic approaches to partition functions or indices ultimately reduce the problem to a matrix model, whose exact solution has not been established yet. The exactness of the $3 \mathrm{~d}-3 \mathrm{~d}$ correspondence becomes therefore a crucial advantage, particularly in the analysis of subleading orders.

More precisely, in this manuscript we present a computation of the superconformal index of the field theories, and show that it produces the entropy functions that generate the entropy of dual rotating asymptotically $\mathrm{AdS}_{4}$ black holes through a Legendre transform. We also compute the one-loop effective action in 11d supergravity for M5-branes wrapped on the hyperbolic 3-space. We then match the gravity result with the field theory answer. In particular, we demonstrate that the agreement persists even when we consider hyperbolic 3-manifolds that are more general than those previously considered in the literature, including non-trivial first Betti number $b_{1}$. Our subleading computation has the potential to distinguish between various supersymmetric observables that have been shown to yield the same entropy function at leading order.

Finally, we use the $3 \mathrm{~d}-3 \mathrm{~d}$ correspondence to evaluate the refined topologically twisted index, and find that it vanishes in the large $N$ limit. This matches with the fact that there are no BPS rotating magnetically-charged black holes with spherical horizon topology in $4 \mathrm{~d} \mathcal{N}=2$ minimal gauged supergravity. In fact, we provide evidence of an even stronger result: that the refined topologically twisted index exactly vanishes at finite $N$. This result is a mathematical curiosity that has been previously entertained. It translates to a very non-trivial property of Chern-Simons perturbative invariants of hyperbolic three-manifolds, 
that would be interesting to mathematically explore. ${ }^{1}$ We will offer a physical argument for such a vanishing.

The rest of the paper is organized as follows. In section 2 we provide a $3 \mathrm{~d}-3 \mathrm{~d}$ perspective on various indices computed in the corresponding $3 \mathrm{~d} \mathcal{N}=2$ superconformal field theories. In section 3 we compute the indices in the large $N$ limit. Section 4 discusses properties of the supergravity description of wrapped M5-branes on hyperbolic 3-manifolds, including some of the relevant solutions. Some details regarding the one-loop correction to terms logarithmic in $N$, from the supergravity point of view, are presented in section 5 . We conclude in section 6 where we also highlight a number of interesting problems. In appendix A we provide explicit examples of state-integral models for complex Chern-Simons theory, from which perturbative Chern-Simons invariants can be computed.

Note added. While we were preparing this manuscript for submission, we received [30] which has some overlap regarding the leading behavior of the superconformal index.

\section{$23 \mathrm{~d}-3 \mathrm{~d}$ relations for $3 \mathrm{~d}$ indices}

We begin by studying the 3d-3d relations for three types of $3 \mathrm{~d}$ supersymmetric indices: the superconformal index, the refined topologically twisted index on $S^{2}$, and the topologically twisted index on a general Riemann surface $\Sigma_{g}$ of genus $g$. Schematically, the 3d-3d relations take the following form:

A supersymmetric index of the $3 \mathrm{~d} \mathcal{N}=2 \mathcal{T}_{N}\left[M_{3}\right]$ theory

$$
=\text { An invariant of } \operatorname{SL}(N, \mathbb{C}) \text { Chern-Simons theory on } M_{3} \text {. }
$$

In subsections 2.1 and 2.2 we will explain the basic definitions and properties of the two quantum field theories - a supersymmetric theory and a topological theory, respectively - appearing in the relations. Then, in subsection 2.3 we will give precise formulations of the $3 \mathrm{~d}-3 \mathrm{~d}$ relations for the three types of indices. The part on the superconformal index will basically be a review of previous work [28, 31, 32], with some additional clarifications. On the other hand, we will propose a $3 \mathrm{~d}-3 \mathrm{~d}$ relation for the refined version of the twisted index, with non-trivial supporting evidence. Motivated by that, we will also propose a $3 \mathrm{~d}-3 \mathrm{~d}$ relation for the twisted index on general Riemann surfaces, thus generalizing previous work [33] and covering general hyperbolic 3-manifolds $M_{3}$. Finally, in subsection 2.4 we will provide non-trivial consistency checks for the proposed $3 \mathrm{~d}-3 \mathrm{~d}$ relations, confirming the expected integral properties of the various indices.

\section{$2.13 \mathrm{~d} \mathcal{T}_{N}\left[M_{3}\right]$ theory}

Let us first recall the definition and basic properties of the $3 \mathrm{~d} \mathcal{T}_{N}\left[M_{3}\right]$ theory appearing on the left-hand-side (1.h.s.) of the 3d-3d relation (2.1). We define

$$
\begin{aligned}
& \mathcal{T}_{N}\left[M_{3}\right]:=(\text { the } 3 \mathrm{~d} \mathcal{N}=2 \text { superconformal field theory obtained from a twisted } \\
& \text { compactification of the } \left.6 \mathrm{~d} \mathcal{N}=(2,0) \text { theory of type } A_{N-1} \text { on } M_{3}\right) \text {, } \\
& M_{3}=(\text { a closed hyperbolic } 3 \text {-manifold })=\mathbb{H}^{3} / \Gamma \text {. }
\end{aligned}
$$

\footnotetext{
${ }^{1}$ Some work in this direction is in progress [29].
} 
We use an $\mathrm{SO}(3)$ subgroup of the $\mathrm{SO}(5)$ R-symmetry of the $6 \mathrm{~d}$ theory to perform partial topological twist along $M_{3}$. The resulting $3 \mathrm{~d}$ theory $\mathcal{T}_{N}\left[M_{3}\right]$ has $3 \mathrm{~d} \mathcal{N}=2$ supersymmetry, with no (non-R) flavor symmetries at sufficiently large $N$. The $\mathrm{U}(1) \cong \mathrm{SO}(2)$ R-symmetry of the $3 \mathrm{~d}$ theory comes from the $\mathrm{SO}(2) \subset \mathrm{SO}(2) \times \mathrm{SO}(3) \subset \mathrm{SO}(5)$ subgroup of the $6 \mathrm{~d}$ R-symmetry, and thus its charges $R$ are quantized:

$$
R \in \mathbb{Z} \quad \text { in } \mathcal{T}_{N}\left[M_{3}\right] \text { theory } .
$$

At small $N$ there could be an accidental flavor symmetry in $\mathcal{T}_{N}\left[M_{3}\right]$ as studied in [34, 35]. In that case, the infrared (IR) superconformal R-symmetry could be different from the Rsymmetry originated from $\mathrm{SO}(2) \subset \mathrm{SO}(5)$. The field theoretic construction of $\mathcal{T}_{N}\left[M_{3}\right]$ was proposed in [27, 35, 36] based on a Dehn-surgery representation of $M_{3}$ using a link $L \subset S^{3}$ and an ideal triangulation of the link complement $S^{3} \backslash L$. The theory $\mathcal{T}_{N}\left[M_{3}\right]$ is expected to be independent of the choice of Dehn-surgery representation and ideal triangulation. ${ }^{2}$ Such an invariance gives a geometric understanding of certain dualities among $3 \mathrm{~d} \mathcal{N}=2$ gauge theories.

\section{$2.2 \operatorname{SL}(N, \mathbb{C})$ Chern-Simons theory on $M_{3}$}

The most general action for $\operatorname{SL}(N, \mathbb{C})$ Chern-Simons theory on $M_{3}$ is given by

$$
\begin{aligned}
S_{k, \sigma}\left[\mathcal{A}, \widetilde{\mathcal{A}} ; M_{3}\right] & =\frac{(k+i \sigma)}{8 \pi} \int_{M_{3}} \operatorname{Tr}\left(\mathcal{A} \wedge d \mathcal{A}+\frac{2}{3} \mathcal{A}^{3}\right)+\frac{(k-i \sigma)}{8 \pi} \int_{M_{3}} \operatorname{Tr}\left(\widetilde{\mathcal{A}} \wedge d \widetilde{\mathcal{A}}+\frac{2}{3} \widetilde{\mathcal{A}}^{3}\right) \\
& =\frac{2 \pi i}{\hbar} \operatorname{CS}\left[\mathcal{A} ; M_{3}\right]+\frac{2 \pi i}{\tilde{\hbar}} \operatorname{CS}\left[\widetilde{\mathcal{A}} ; M_{3}\right] .
\end{aligned}
$$

We have introduced (anti-)holomorphic couplings and the Chern-Simons functional:

$$
\begin{aligned}
\hbar & :=\frac{4 \pi i}{k+i \sigma}, & \tilde{\hbar}:=\frac{4 \pi i}{k-i \sigma}, \\
\operatorname{CS}\left[\mathcal{A} ; M_{3}\right] & :=\frac{1}{4 \pi} \int_{M_{3}} \operatorname{Tr}\left(\mathcal{A} \wedge d \mathcal{A}+\frac{2}{3} \mathcal{A}^{3}\right) . &
\end{aligned}
$$

For gauge invariance and unitarity of the theory, it is required that

$$
k \in \mathbb{Z}, \quad \sigma \in \mathbb{R} \text { or } i \mathbb{R} .
$$

The $\operatorname{SL}(N, \mathbb{C})$ Chern-Simons partition function is defined by the following path-integral:

$$
\begin{aligned}
\mathcal{Z}_{k, \sigma}^{\mathrm{SL}(N, \mathbb{C})}\left[M_{3} ;\left\{n_{\alpha \beta}\right\}\right] & =\int_{\Gamma\left(\left\{n_{\alpha \beta}\right\}\right)} \frac{[D \mathcal{A}][D \widetilde{\mathcal{A}}]}{(\text { gauge })} e^{i S_{k, \sigma}\left[\mathcal{A}, \widetilde{A} ; M_{3}\right]}, \\
\Gamma\left(\left\{n_{\alpha \beta}\right\}\right) & :=\sum_{\mathcal{A}^{\alpha}, \widetilde{\mathcal{A}}^{\beta} \in \chi\left(M_{3} ; N\right)} n_{\alpha, \beta} \mathcal{C}^{\alpha}(\mathcal{A}) \times \mathcal{C}^{\beta}(\widetilde{\mathcal{A}}),
\end{aligned}
$$

where $\chi\left(M_{3} ; N\right)$ represents

$$
\chi\left(M_{3} ; N\right):=\left(\text { set of gauge-inequivalent } \operatorname{SL}(N, \mathbb{C}) \text { flat connections on } M_{3}\right) .
$$

\footnotetext{
${ }^{2}$ This approach requires that the link complement be hyperbolic.
} 
Moreover, $\mathcal{C}^{\alpha}(\mathcal{A})$ denotes the absolutely convergent integration cycle (Lefschetz thimble) in the configuration space of gauge fields $\mathcal{A}$, associated to a flat connection $\mathcal{A}^{\alpha}$. (See, for example, [37] for details on the integration cycle $\mathcal{C}^{\alpha}(\mathcal{A})$.) Thus, to specify a quantum $\mathrm{SL}(N, \mathbb{C})$ Chern-Simons theory, we need to specify the choice of a consistent integration cycle $\left\{n_{\alpha \beta}\right\}$ as well as the CS levels $(k, \sigma)$.

The partition function can be factorized as

$$
\mathcal{Z}_{k, \sigma}^{\mathrm{SL}(N, \mathbb{C})}\left[M_{3} ;\left\{n_{\alpha \beta}\right\}\right]=\sum_{\mathcal{A}^{\alpha}, \widetilde{\mathcal{A}}^{\beta} \in \chi\left(M_{3} ; N\right)} \frac{1}{|\operatorname{Stab}(\alpha, \beta)|} n_{\alpha, \beta} B_{M_{3}}^{\alpha}(q ; N) B_{M_{3}}^{\beta}(\tilde{q} ; N) .
$$

Here the "holomorphic blocks" $B^{\alpha}(q)$ are defined as

$$
B_{M_{3}}^{\alpha}\left(q:=e^{\hbar} ; N\right)=\int_{\mathcal{C}^{\alpha}(\mathcal{A})} \frac{[D \mathcal{A}]}{(\text { gauge })} e^{-\frac{2 \pi}{\hbar} \operatorname{CS}\left[\mathcal{A} ; M_{3}\right]}
$$

We defined the exponentiated holomorphic and anti-holomorphic couplings as

$$
q:=e^{\hbar}=e^{\frac{4 \pi i}{k+i \sigma}}, \quad \tilde{q}:=e^{\tilde{\hbar}}=e^{\frac{4 \pi i}{k-i \sigma}} .
$$

The prefactor $|\operatorname{Stab}(\alpha, \beta)|$ is the volume of the stabilizer of $\left(\mathcal{A}^{\alpha}, \widetilde{\mathcal{A}}^{\beta}\right)$, i.e., the volume of the subgroup of the gauge group that preserves the flat connections. In an asymptotic $\hbar \rightarrow 0$ limit, the holomorphic blocks $B^{\alpha}$ can be perturbatively expanded:

$$
B_{M_{3}}^{\alpha}(q) \stackrel{\hbar \rightarrow 0}{\longrightarrow} \exp \left(\frac{1}{\hbar} S_{0}^{\alpha}\left(M_{3} ; N\right)+S_{1}^{\alpha}\left(M_{3} ; N\right)+\ldots+\hbar^{n-1} S_{n}^{\alpha}\left(M_{3} ; N\right)+\ldots\right)
$$

For example

$$
S_{0}^{\alpha}=-2 \pi \operatorname{CS}\left[\mathcal{A}^{\alpha} ; M_{3}\right], \quad S_{1}^{\alpha}=-\frac{1}{2} \operatorname{Tor}\left[\mathcal{A}^{\alpha} ; M_{3}\right]
$$

where $\operatorname{Tor}\left[\mathcal{A}^{\alpha} ; M_{3}\right]$ is the analytic Ray-Singer torsion twisted by the flat connection $\mathcal{A}^{\alpha}$ defined as follows [38, 39]:

$$
\operatorname{Tor}\left[\mathcal{A}^{\alpha} ; M_{3}\right]:=\frac{\left[\operatorname{det}^{\prime} \Delta_{1}\left(\mathcal{A}^{\alpha}\right)\right]^{1 / 4}}{\left[\operatorname{det}^{\prime} \Delta_{0}\left(\mathcal{A}^{\alpha}\right)\right]^{3 / 4}}
$$

Above, $\Delta_{n}\left(\mathcal{A}^{\alpha}\right)$ is the Laplacian acting on $\mathfrak{s l}(N, \mathbb{C})$-valued $n$-forms twisted by a flat connection $\mathcal{A}^{\alpha}$ :

$$
\Delta_{n}(\mathcal{A})=d_{\mathcal{A}} * d_{\mathcal{A}} *+* d_{\mathcal{A}} * d_{\mathcal{A}}, \quad d_{\mathcal{A}}=d+\mathcal{A} \wedge
$$

Then, $\operatorname{det}^{\prime} \Delta$ denotes the zeta-function regularized product of non-zero eigenvalues of $\Delta$. To define the Laplacian, one needs to introduce a smooth metric on $M_{3}$. The torsion is however independent of the metric choice and is a topological invariant. 


\section{$2.3 \quad 3 d-3 d$ relations}

We consider three types of supersymmetric indices of $3 \mathrm{~d} \mathcal{N}=2$ gauge theories: the superconformal index, the refined topologically twisted index on $S^{2}$, and twisted index on general closed Riemann surfaces $\Sigma_{g}$ of genus $g$. Let us define

$$
\begin{aligned}
& \mathcal{I}_{\text {sci }}\left(q ; \mathcal{T}_{N}\left[M_{3}\right]\right)=\left(\text { Superconformal index of } \mathcal{T}_{N}\left[M_{3}\right] \text { theory on } S^{2}\right) \\
& :=\operatorname{Tr}_{\mathcal{H}_{\mathrm{sci}}\left(S^{2}\right)}(-1)^{R} q^{\frac{R}{2}+j_{3}}, \\
& \mathcal{I}_{\text {top }}\left(q ; \mathcal{T}_{N}\left[M_{3}\right]\right)=\left(\text { Refined topologically twisted index of } \mathcal{T}_{N}\left[M_{3}\right] \text { theory on } S^{2}\right) \\
& :=\operatorname{Tr}_{\mathcal{H}_{\text {top }}\left(S^{2}\right)}(-1)^{R} q^{j_{3}} \\
& \mathcal{I}_{\Sigma_{g}}\left(\mathcal{T}_{N}\left[M_{3}\right]\right)=\left(\text { Topologically twisted index of } \mathcal{T}_{N}\left[M_{3}\right] \text { theory on } \Sigma_{g}\right) \\
& :=\operatorname{Tr}_{\mathcal{H}_{\text {top }}\left(\Sigma_{g}\right)}(-1)^{R} \text {. }
\end{aligned}
$$

Here $R$ is the R-charge corresponding to the compact $\mathrm{SO}(2)$ R-symmetry subgroup of $\mathrm{SO}(5)$, while $j_{3}$ is the Cartan generator of the $\mathfrak{s u}(2) \cong \mathfrak{s o}(3)$ isometry of $S^{2}$. To produce protected indices, instead of $(-1)^{F}$ or $(-1)^{2 j_{3}}$ we use $(-1)^{R}$ which typically appears in the context of $3 \mathrm{~d}-3 \mathrm{~d}$ correspondences. Then $\mathcal{H}_{\text {sci }}\left(S^{2}\right)$ is the radially-quantized Hilbert space of $\mathcal{T}_{N}\left[M_{3}\right]$, whose elements are linear combinations of local operators. Only $1 / 4$ BPS operators satisfying $\Delta=R+j_{3}$, where $\Delta$ is the conformal dimension, contribute to the index. On the other hand, $\mathcal{H}_{\text {top }}\left(\Sigma_{g}\right)$ is the Hilbert space of the topologically twisted $\mathcal{T}_{N}\left[M_{3}\right]$ theory on a closed Riemann surface $\Sigma_{g}$. In the topological twisting, we turn on a background gauge field $A_{R}^{\text {background }}$ coupled to the $\mathrm{SO}(2)$ R-symmetry along $\Sigma_{g}$ in such a way that

$$
A_{R}^{\text {background }}=\frac{1}{2} \omega\left(\Sigma_{g}\right) \Rightarrow \frac{1}{2 \pi} \int_{\Sigma_{g}} d A_{R}^{\text {backgrond }}=(1-g)
$$

Here $\omega\left(\Sigma_{g}\right)$ is the spin-connection on $\Sigma_{g}$. Due to the background magnetic flux, the following Dirac quantization conditions are required:

$$
(g-1) R(\mathcal{O}) \in \mathbb{Z}
$$

for all scalar gauge-invariant operators $\mathcal{O}$ in the theory. Thanks to (2.3), the quantization condition is automatically satisfied for $\mathcal{T}_{N}\left[M_{3}\right]$ theories and we can consider the twisted index for arbitrary $g$. When $g=0$, i.e., for $\Sigma_{g}=S^{2}$, there is an additional $\mathfrak{s u}(2)$ rotational symmetry in the topologically twisted background, and we can refine the twisted index by turning on a fugacity $q$ associated to the Cartan generator $j_{3}$ of $\mathfrak{s u}(2)$. As typical for a Witten index, the twisted index only gets contributions from ground states in $\mathcal{H}_{\text {top }}\left(\Sigma_{g}\right)$, whose number is finite. 


\subsubsection{Superconformal index}

The $3 \mathrm{~d}-3 \mathrm{~d}$ relation for the superconformal index was studied in [28, 31, 32, 40, 41], and the following proposal was made:

$$
\begin{aligned}
\mathcal{I}_{\text {sci }}\left(q ; \mathcal{T}_{N}\left[M_{3}\right]\right) & =\mathcal{Z}_{k=0, \sigma=\frac{2 \pi}{\log q}}^{\mathrm{SL}(N, \mathbb{C})}\left[M_{3},\left\{n_{\alpha \beta}=\delta_{\alpha \bar{\beta}}\right\}\right] \\
& =\sum_{\mathcal{A}^{\alpha} \in \chi\left(M_{3} ; N\right)} \frac{1}{|\operatorname{Stab}(\alpha)|} B_{M_{3}}^{\alpha}(q ; N) B_{M_{3}}^{\bar{\alpha}}\left(q^{-1} ; N\right) \\
& =\sum_{\mathcal{A}^{\alpha} \in \chi_{\text {irred }}\left(M_{3} ; N\right)} \frac{1}{|\operatorname{Stab}(\alpha)|} B_{M_{3}}^{\alpha}(q ; N) B_{M_{3}}^{\bar{\alpha}}\left(q^{-1} ; N\right) .
\end{aligned}
$$

For a reducible flat connection $\mathcal{A}^{\alpha}$, its stablizer subgroup is non-compact, $\left|\operatorname{Stab}\left(\mathcal{A}^{\alpha}\right)\right|=\infty$ and thus it does not contribute to the path-integral — see [42, 43] for more detailed discussions on this issue. This is one crucial difference between Chern-Simons theory with compact and non-compact gauge group. Therefore, we only need to sum over a subset $\chi_{\text {irred }}\left(M_{3} ; N\right)$ of flat connections defined as

$\chi_{\text {irred }}\left(M_{3} ; N\right):=\left(\right.$ set of irreducible gauge-inequivalent $\operatorname{SL}(N, \mathbb{C})$ flat connections on $\left.M_{3}\right)$.

Alternatively, we can take the point of view advocated, e.g., in [35, 42] that the theory $\mathcal{T}_{N}\left[M_{3}\right]$ describes only part of the moduli space of $N$ M5-branes on $M_{3}$. This moduli subspace is essentially a Higgs branch (as the theories $\mathcal{T}_{N}\left[M_{3}\right]$ have Higgs but no Coulomb branch) and its points correspond the irreducible $\mathrm{SL}(N, \mathbb{C})$ flat connections on $M_{3}$, leading to $\chi_{\text {irred }}\left(M_{3} ; N\right)$.

In (2.19), $\mathcal{A}^{\bar{\alpha}}$ denotes the complex conjugation of $\mathcal{A}^{\alpha}$ :

$$
\mathcal{A}^{\bar{\alpha}}=\left(\mathcal{A}^{\alpha}\right)^{*} .
$$

The 3d-3d relation (2.19) can be further simplified using the following fact:

$$
\begin{array}{ll}
B_{M_{3}}^{\alpha_{1}}(q ; N)=B_{M_{3}}^{\alpha_{2}}(q ; N) & \text { if } \mathcal{A}^{\alpha_{1}} \text { and } \mathcal{A}^{\alpha_{2}} \text { are related to each other by } \\
\text { tensoring with a } \mathbb{Z}_{N} \text { flat-connection } .
\end{array}
$$

Here $\mathbb{Z}_{N}$ is the center of $\operatorname{SL}(N, \mathbb{C})$. Let us explain this tensoring more explicitly. Each $\mathrm{SL}(N, \mathbb{C})$ flat-connection $\mathcal{A}^{\alpha}$ determines a group homomorphism $\rho^{\alpha}$

$$
\rho^{\alpha} \in \operatorname{Hom}\left(\pi_{1}\left(M_{3}\right) \rightarrow \operatorname{SL}(N, \mathbb{C})\right) / \operatorname{SL}(N, \mathbb{C})
$$

up to conjugation. Similarly, a $\mathbb{Z}_{N}$ flat-connection determines a homomorphism $\eta$

$$
\eta \in \operatorname{Hom}\left(\pi_{1}\left(M_{3}\right) \rightarrow \mathbb{Z}_{N}\right)=\operatorname{Hom}\left(H_{1}\left(M_{3}, \mathbb{Z}\right) \rightarrow \mathbb{Z}_{N}\right) .
$$

By tensoring with $\eta$, we can obtain from a flat connection $\mathcal{A}^{\alpha}$ another flat connection $\mathcal{A}^{\eta \otimes \alpha}$ whose $\operatorname{SL}(N, \mathbb{C})$ holonomies are given by

$$
\rho^{\eta \otimes \alpha}(c)=\eta(c) \cdot \rho^{\alpha}(c), \quad \forall c \in \pi_{1}\left(M_{3}\right) .
$$


Here $\cdot$ is the group multiplication in $\operatorname{SL}(N, \mathbb{C})$. Since there are only adjoint fields in $\mathrm{SL}(N, \mathbb{C})$ Chern-Simons theory, the equality in $(2.22)$ holds. More precisely, $(2.25)$ is the action of the $\mathbb{Z}_{N}$ 1-form center symmetry of $\operatorname{SL}(N, \mathbb{C})$ Chern-Simons theory and it leaves the path-integrand invariant. Using (2.22) and the fact that

$$
\left|\operatorname{Stab}\left(\mathcal{A}^{\alpha} \in \chi_{\text {irred }}\left(M_{3} ; N\right)\right)\right|=\mid \text { Center of } \operatorname{SL}(N, \mathbb{C}) \mid=N,
$$

we obtain the following final version of the $3 \mathrm{~d}-3 \mathrm{~d}$ relation for the superconformal index:

$$
\begin{aligned}
\mathcal{I}_{\text {sci }}\left(q ; \mathcal{T}_{N}\left[M_{3}\right]\right) & =\mathcal{Z}_{k=0, \sigma=\frac{2 \pi}{\log q}}^{\mathrm{SL}(N, \mathbb{C})}\left[M_{3},\left\{n_{\alpha \beta}=\delta_{\alpha \bar{\beta}}\right\}\right] \\
& =\frac{\left|\operatorname{Hom}\left[\pi_{1}\left(M_{3}\right) \rightarrow \mathbb{Z}_{N}\right]\right|}{N} \sum_{\left[\mathcal{A}^{\alpha}\right] \in \frac{\chi_{\text {irred }}\left(M_{3} ; N\right)}{\operatorname{Hom}\left[\pi_{1}\left(M_{3}\right) \rightarrow \mathbb{Z}_{N}\right]}} B_{M_{3}}^{\alpha}(q ; N) B_{M_{3}}^{\bar{\alpha}}\left(q^{-1} ; N\right)
\end{aligned}
$$

Here $\left[\mathcal{A}^{\alpha}\right]$ is an equivalence class in $\chi_{\text {irred }}\left(M_{3} ; N\right)$ under the equivalence relation defined by tensoring with $\mathbb{Z}_{N}$ flat connections:

$$
\left[\mathcal{A}^{\alpha}\right]=\left[\mathcal{A}^{\eta \otimes \alpha}\right], \quad \forall \eta \in \operatorname{Hom}\left[\pi_{1}\left(M_{3}\right) \rightarrow \mathbb{Z}_{N}\right] .
$$

\subsubsection{Refined twisted index}

From the factorization property of the refined twisted index $[9,44],{ }^{3}$ we naturally propose the following $3 \mathrm{~d}-3 \mathrm{~d}$ relation:

$$
\begin{aligned}
\mathcal{I}_{\text {top }}\left(q ; \mathcal{T}_{N}\left[M_{3}\right]\right) & =\mathcal{Z}_{k=0, \sigma=\frac{2 \pi}{\log q}}^{\mathrm{SL}(N, \mathbb{C})}\left[M_{3},\left\{n_{\alpha \beta}=\delta_{\alpha \beta}\right\}\right] \\
& =\frac{\left|\operatorname{Hom}\left[\pi_{1}\left(M_{3}\right) \rightarrow \mathbb{Z}_{N}\right]\right|}{N} \sum_{\left[\mathcal{A}^{\alpha}\right] \in \frac{\chi_{\text {irred }}\left(M_{3} ; N\right)}{\operatorname{Hom}\left[\pi_{1}\left(M_{3}\right) \rightarrow \mathbb{Z}_{N}\right]}} B_{M_{3}}^{\alpha}(q ; N) B_{M_{3}}^{\alpha}\left(q^{-1} ; N\right) .
\end{aligned}
$$

This looks similar to (2.27) but, as opposed to the former, this formula has no complex conjugation of the holomorphic blocks. A study of the refined twisted index in the context of the $3 \mathrm{~d}-3 \mathrm{~d}$ correspondence already appeared in [47]. However, the relation we propose is different and complementary to theirs. In [47], the twisted (half-)index was related to homological blocks labelled by Abelian flat connections. On the other hand, we propose a relation with holomorphic blocks labelled by irreducible flat connections.

As an initial consistency check of our proposal, we can obtain the $3 \mathrm{~d}-3 \mathrm{~d}$ relation for the (unrefined) twisted index at $g=0$ put forward in [33], by taking the $q \rightarrow 1$ limit of the

\footnotetext{
${ }^{3}$ The refined index of the tetrahedron $(\Delta)$ theory $\mathcal{T}_{N=2}\left[M_{3}=\Delta\right][27]$ can be written as $B_{\Delta}(q, z) B_{\Delta}(\tilde{q}, \tilde{z})$ with $\tilde{q}=q^{-1}$ and $\tilde{z}=z$. In the $3 \mathrm{~d}-3 \mathrm{~d}$ relation, $q=e^{\frac{4 \pi i}{k+i \sigma}}$ and $\tilde{q}=e^{\frac{4 \pi i}{k-i \sigma}}$. So the relation $\tilde{q}=q^{-1}$ implies $k=0$. Then $z$ and $\tilde{z}$ parametrize boundary $\operatorname{SL}(2, \mathbb{C})$ holonomies of the gauge fields $\mathcal{A}$ and $\widetilde{\mathcal{A}}$, respectively. Thus the relation $z=\tilde{z}$ implies $n_{\alpha \beta}=\delta_{\alpha \beta}$. From the point of view of the supersymmetric field theory, factorization of the partition function can be understood in terms of Higgs branch localization to vortex and anti-vortex partition functions [45, 46].
} 
refined index:

$$
\begin{aligned}
& \left.\mathcal{I}_{\text {top }}\left(q ; \mathcal{T}_{N}\left[M_{3}\right]\right)\right|_{q \rightarrow 1} \\
& =\left.\frac{\left|\operatorname{Hom}\left[\pi_{1}\left(M_{3}\right) \rightarrow \mathbb{Z}_{N}\right]\right|}{N} \sum_{\left[\mathcal{A}^{\alpha}\right] \in \frac{\chi_{\text {irred }\left(M_{3} ; N\right)}}{\operatorname{Hom}\left[\pi_{1}\left(M_{3}\right) \rightarrow \mathbb{Z}_{N}\right]}} B_{M_{3}}^{\alpha}(q ; N) B_{M_{3}}^{\alpha}\left(q^{-1} ; N\right)\right|_{q \rightarrow 1} \\
& =\left.\frac{\left|\operatorname{Hom}\left[\pi_{1}\left(M_{3}\right) \rightarrow \mathbb{Z}_{N}\right]\right|}{N} \sum_{\left[\mathcal{A}^{\alpha}\right]} \exp \left(\sum_{n=0}^{\infty} \hbar^{n-1} S_{n}^{\alpha}\right) \exp \left(\sum_{n=0}^{\infty}(-\hbar)^{n-1} S_{n}^{\alpha}\right)\right|_{\hbar \rightarrow 0} \\
& =\frac{\left|\operatorname{Hom}\left[\pi_{1}\left(M_{3}\right) \rightarrow \mathbb{Z}_{N}\right]\right|}{N} \sum_{\left[\mathcal{A}^{\alpha}\right]} \exp \left(2 S_{1}^{\alpha}\left(M_{3} ; N\right)\right) .
\end{aligned}
$$

Notice that only the 1-loop part contributes in the unrefined limit. The $3 \mathrm{~d}-3 \mathrm{~d}$ relation for twisted indices proposed in [33] is

$$
\mathcal{I}_{\Sigma_{g}}\left(\mathcal{T}_{N}\left[M_{3}\right]\right)=\sum_{\alpha \in \chi_{\text {irred }}\left(M_{3} ; N\right)}\left(N \times \exp \left(-2 S_{1}^{\alpha}\right)\right)^{g-1}
$$

for $M_{3}$ with trivial $H_{1}\left(M_{3}, \mathbb{Z}_{N}\right)$.

Such a relation was indirectly derived by combining several recent technical developments: i) field theoretic constructions of $\mathcal{T}_{N}\left[M_{3}\right][27,35,36]$; ii) field theory localization formulas of twisted indices [9, 48, 49]; iii) mathematical tools [50-52] for computing the Ray-Singer torsion $-2 S_{1}^{\alpha}$ from state-integral models for $\mathrm{SL}(N, \mathbb{C})$ Chern-Simons theory. The $3 \mathrm{~d}-3 \mathrm{~d}$ relation (2.31) with $g=0$ precisely matches the $q \rightarrow 1$ limit (2.30) of the proposed $3 \mathrm{~d}-3 \mathrm{~d}$ relation (2.29).

\subsubsection{Twisted indices}

Let us compare the $3 \mathrm{~d}-3 \mathrm{~d}$ relation (2.29) with the following universal expression for the twisted index $\mathcal{I}_{\Sigma_{g=0}}$ of general $3 \mathrm{~d} \mathcal{N}=2$ theories $\mathcal{T}$ :

$$
\mathcal{I}_{\Sigma_{g=0}}(\mathcal{T})=\sum_{\alpha \in \mathcal{S}_{\mathrm{BE}}(\mathcal{T})}\left(\mathcal{H}_{\alpha}\right)^{-1}
$$

Here $\mathcal{S}_{\mathrm{BE}}(\mathcal{T})$ denotes the set of vacua ${ }^{4}$ of the $3 \mathrm{~d} \mathcal{N}=2$ theory obtained by extremizing the effective twisted superpotential on $\mathbb{R}^{2} \times S^{1}$ after summing all one-loop contributions from infinitely many massive Kaluza-Klein modes along $S^{1}$. The order of the set is equal to the Witten index $[53,54]$ of the $3 \mathrm{~d}$ theory

$$
\left|\mathcal{S}_{\mathrm{BE}}(\mathcal{T})\right|=\mid \text { Witten index of } \mathcal{T} \mid
$$

On the other hand, $\mathcal{H}_{\alpha}$ is the so-called handle-gluing operator.

\footnotetext{
${ }^{4}$ The notation BE stands for "Bethe Equations", because the 2d F-term equations from the effective twisted superpotential look similar to Bethe ansatz equations.
} 
We naturally conjecture that

$$
\begin{aligned}
\mathcal{S}_{\mathrm{BE}}(\mathcal{T}) & =\frac{\chi_{\text {irred }}\left(M_{3} ; N\right)}{\operatorname{Hom}\left[\pi_{1}\left(M_{3}\right) \rightarrow \mathbb{Z}_{N}\right]}, \\
\mathcal{H}_{\alpha} & =\frac{N}{\left|\operatorname{Hom}\left[\pi_{1}\left(M_{3}\right) \rightarrow \mathbb{Z}_{N}\right]\right|} \exp \left(-2 S_{1}^{\alpha}\left(M_{3} ; N\right)\right) .
\end{aligned}
$$

This identification leads to the following generalized 3d-3d relation for twisted indices:

$$
\mathcal{I}_{\Sigma_{g}}\left(\mathcal{T}_{N}\left[M_{3}\right]\right)=\sum_{\left[\mathcal{A}^{\alpha}\right] \in \frac{\chi_{\text {irred }}\left(M_{3} ; N\right)}{\operatorname{Hom}\left[\pi_{1}\left(M_{3}\right) \rightarrow \mathbb{Z}_{N}\right]}}\left(\frac{N}{\left|\operatorname{Hom}\left[\pi_{1}\left(M_{3}\right) \rightarrow \mathbb{Z}_{N}\right]\right|} \exp \left(-2 S_{1}^{\alpha}\right)\right)^{g-1}
$$

for arbitrary closed hyperbolic 3-manifolds $M_{3}$.

While in [33] the $3 \mathrm{~d}$-3d relation was proposed for 3 -manifolds with vanishing $H_{1}\left(M_{3}, \mathbb{Z}_{N}\right)$, here we generalize it to arbitrary closed hyperbolic 3-manifolds.

\subsection{Integrality of indices at finite $N$}

To test our proposals, in this subsection we verify a few integrality properties that the indices must satisfy.

\subsubsection{Vanishing of the refined twisted index}

Since the $R$-charge of $\mathcal{T}_{N}\left[M_{3}\right]$ is quantized as in (2.3) and $j_{3} \in \frac{1}{2} \mathbb{Z}$, we expect that

$$
\mathcal{I}_{\text {top }}\left(q ; \mathcal{T}_{N}\left[M_{3}\right]\right) \in \mathbb{Z}\left[q^{1 / 2}, q^{-1 / 2}\right],
$$

namely that the refined twisted index is a finite Laurent series in $q^{1 / 2}$ with integer coefficients. Indeed, the (refined) twisted index is a Witten index, and it only gets contributions from ground states in the twisted Hilbert space $\mathcal{H}_{\text {top }}\left(S^{2}\right)$ which is finite. Since the index is a finite Laurent polynomial in $q^{1 / 2}$, it converges in the limit $q=e^{\hbar} \rightarrow 1$ and we can systematically study it using perturbative methods in complex Chern-Simons theory. The 3d-3d relation (2.29) then translates this rather obvious statement into the following highly non-trivial prediction on the perturbative invariants $\left\{S_{n}\left(M_{3} ; N\right)\right\}$ :

$$
\begin{array}{r}
\frac{\left|\operatorname{Hom}\left[\pi_{1}\left(M_{3}\right) \rightarrow \mathbb{Z}_{N}\right]\right|}{N} \sum_{\substack{\left.\mathcal{A}^{\alpha}\right] \in \frac{\chi_{\text {irred }}\left(M_{3} ; N\right)}{\operatorname{Hom}\left[\pi_{1}\left(M_{3}\right) \rightarrow \mathbb{Z}_{N}\right]} \\
\in}} \exp \left(2 \sum_{n=0}^{\infty} \hbar^{2 n} S_{2 n+1}^{\alpha}\left(M_{3} ; N\right)\right) \\
\in \mathbb{Z}\left[e^{\hbar / 2}, e^{-\hbar / 2}\right] \quad \text { as a formal expansion in } \hbar .
\end{array}
$$

In the following we will check that, in fact, an even stronger statement holds true: the 1.h.s. of (2.37) actually vanishes for many closed hyperbolic 3-manifolds $M_{3}$ (at least up to some high power of $\hbar)$.

We can offer the following physical argument for such a vanishing. The setup is $N$ M5-branes on $S^{2} \times S^{1} \times M_{3}$. The compactification on $S^{2}$ [55] produces a peculiar class $\mathcal{S}$ theory with empty Coulomb branch but non-trivial Higgs branch. This can be understood 
by further compactification on $S^{1}$ : the $3 \mathrm{~d}$ mirror is $3 \mathrm{~d} \mathcal{N}=4 \mathrm{SU}(N) \mathrm{SYM}$ [56], which has hyper-Kähler Coulomb branch and empty Higgs branch. However, assuming that the theory $\mathcal{T}_{N}\left[M_{3}\right]$ only captures a branch of the moduli space of M5-branes, which in this case corresponds to the Coulomb branch of the class $\mathcal{S}$ theory [57], the fact that such a branch is empty implies that the twisted index vanishes.

Example: $\boldsymbol{M}_{\mathbf{3}}=\left(\boldsymbol{S}^{\mathbf{3}} \backslash \mathbf{4}_{1}\right)_{P / Q=5}$ and $\boldsymbol{N}=\mathbf{2}$. We refer to (A.1) for our notation of Dehn-surgery representation of closed 3-manifolds. Here $\mathbf{4}_{1}$ is the figure-eight knot. This is known as Meyerhoff 3-manifold, and its hyperbolic volume is approximately

$$
\operatorname{vol}\left(M_{3}\right) \simeq 0.981369 \text {. }
$$

The corresponding $3 \mathrm{~d}$ gauge theory for $N=2$ is [34]

$$
\mathcal{T}_{N=2}\left[M_{3}\right]=\left(\mathrm{U}(1)_{k=-7 / 2} \text { coupled to a single chiral } \Phi \text { of charge }+1\right) .
$$

The perturbative invariants are:

$$
\begin{aligned}
S_{0}\left(\left(S^{3} \backslash \mathbf{4}_{1}\right)_{5} ; N=2\right)= & \operatorname{Li}_{2}\left(e^{-Z}\right)+i \pi\left(1+2 \ell_{z}\right) Z-\frac{3}{2} Z^{2}, \\
S_{1}\left(\left(S^{3} \backslash \mathbf{4}_{1}\right)_{5} ; N=2\right)= & \log z-\frac{1}{2} \log (3 z-4)+\frac{1}{2} \log 2, \\
S_{2}\left(\left(S^{3} \backslash \mathbf{4}_{1}\right)_{5} ; N=2\right)= & \frac{27 z^{3}-54 z^{2}+92 z-64}{24(3 z-4)^{3}}, \\
S_{3}\left(\left(S^{3} \backslash \mathbf{4}_{1}\right)_{5} ; N=2\right)= & \frac{5 z\left(21 z^{3}-49 z^{2}+36 z-8\right)}{2(4-3 z)^{6}}, \\
S_{4}\left(\left(S^{3} \backslash \mathbf{4}_{1}\right)_{5} ; N=2\right)= & \frac{z\left(-19683 z^{7}+318087 z^{6}+75762 z^{5}-2103318 z^{4}+2881056 z^{3}\right)}{720(3 z-4)^{9}} \\
& +\frac{z\left(-1056608 z^{2}-265152 z+169856\right)}{720(3 z-4)^{9}}, \\
S_{5}\left(\left(S^{3} \backslash \mathbf{4}_{1}\right)_{5} ; N=2\right)= & \frac{z\left(-11907 z^{9}+671895 z^{8}-1057914 z^{7}-2768940 z^{6}+8509962 z^{5}\right)}{24(4-3 z)^{12}} \\
& +\frac{z\left(-7816248 z^{4}+2119680 z^{3}+809536 z^{2}-511872 z+55808\right)}{24(4-3 z)^{12}} .
\end{aligned}
$$

Here $z:=e^{Z}$ and $\ell_{z} \in \mathbb{Z}$ take different values on each irreducible flat connection. There are 4 irreducible $\mathrm{SL}(2, \mathbb{C})$ flat connections on $M_{3}$, which correspond to the following 4 Bethe vacua:

$$
\exp \left(\partial_{Z} S_{0}\right)=1 \quad \Rightarrow \quad \mathcal{S}_{\mathrm{BE}}\left(\mathcal{T}_{N=2}\left[M_{3}=\left(S^{3} \backslash \mathbf{4}_{1}\right)_{5}\right]\right)=\left\{z \mid \frac{1-z}{z^{4}}=1\right\} .
$$

The manifold $M_{3}$ has vanishing $H_{1}\left(M_{3}, \mathbb{Z}_{2}\right)$ and there are no $\mathbb{Z}_{2}$ flat connections. This is compatibile with the Witten index computation [54]:

$$
\begin{aligned}
& \text { Witten index of }\left(\mathrm{U}(1)_{k}+\text { fundamental } \Phi\right)=|k|+1 / 2 \\
& \Rightarrow \text { Witten index of } \mathcal{T}_{N=2}\left[M_{3}=\left(S^{3} \backslash \mathbf{4}_{1}\right)_{5}\right]=4 .
\end{aligned}
$$


For each solution to the Bethe vacua equation, there exists a solution $Z$ satisfying $\partial_{Z} S_{0}=0$ for a proper choice of $\ell_{z} \in \mathbb{Z}$ : this fixes $\ell_{z}$. Except for the classical part $S_{0}$, the perturbative invariants are independent of the choice of $\ell_{z}$. The numerical values of $Z, \ell_{z}$ on the Bethe vacua and their classical actions are

$$
\begin{array}{rrr}
\mathcal{A}^{\overline{\mathrm{geom}}}: Z=0.061412+1.33528 i & \left(\ell_{z}=0\right), & S_{0}=-1.52067-0.981369 i, \\
\mathcal{A}^{\text {geom }}: Z=0.061412-1.33528 i & \left(\ell_{z}=-1\right), & S_{0}=-1.52067+0.981369 i, \\
\mathcal{A}^{3 \mathrm{rd}}: Z=0.199461+\pi i & \left(\ell_{z}=1\right), & S_{0}=-15.5579, \\
\mathcal{A}^{4 \mathrm{th}}: Z=-0.322285 & \left(\ell_{z}=-1\right), & S_{0}=2.14992 .
\end{array}
$$

Note that $\operatorname{Im}\left[S_{0}^{\text {geom }}\right]=-\operatorname{Im}\left[S_{0}^{\overline{\text { geom }}}\right]=\operatorname{vol}\left(M_{3}\right)$. The $\mathcal{A}^{\text {3rd }}$ and $\mathcal{A}^{\text {th }}$ are irreducible flat connections in $\mathrm{SU}(2) \subset \mathrm{SL}(2, \mathbb{C})$ and have vanishing $\operatorname{Im}\left[S_{0}\right]$. Using the perturbative invariants (recall $q=e^{\hbar}$, one can check that

$$
\begin{aligned}
& \mathcal{I}_{\text {top }}\left(q ; \mathcal{T}_{N=2}\left[M_{3}=\left(S^{3} \backslash \mathbf{4}_{1}\right)_{5}\right]\right) \\
& =\left.\frac{1}{2} \sum_{\frac{1-z}{z^{4}}=1} \exp \left(2 S_{1}\left(M_{3} ; N\right)+2 S_{3}\left(M_{3} ; N\right) \hbar^{2}+2 S_{5}\left(M_{3} ; N\right) \hbar^{4}+\mathcal{O}\left(\hbar^{6}\right)\right)\right|_{M_{3}=\left(S^{3} \backslash \mathbf{4}_{1}\right)_{5}} ^{N=2} \\
& =0+\mathcal{O}\left(\hbar^{6}\right)
\end{aligned}
$$

Surprisingly, the refined twisted index vanishes (at least up to a certain power of $\hbar$ ) in a highly non-trivial way. We have checked that the vanishing persists up to $\mathcal{O}\left(\hbar^{10}\right)$.

Example: $M_{3}=($ Weeks manifold $):=\left(S^{\mathbf{3}} \backslash \mathbf{5}_{1}^{\mathbf{2}}\right)_{P_{1} / Q_{1}=-5, P_{2} / Q_{2}=-5 / 2}$. Here $5_{1}^{2}$ is the Whitehead link. This manifold is the smallest orientable closed hyperbolic 3 -manifold [58], and its volume is

$$
\operatorname{vol}\left(M_{3}\right) \simeq 0.942707
$$

The corresponding $3 \mathrm{~d}$ gauge theory for $N=2$ is [34]

$$
\mathcal{T}_{N=2}\left[M_{3}\right]=\left(\mathrm{U}(1)_{k=-5 / 2} \text { coupled to a single chiral } \Phi \text { of charge }+1\right) .
$$

The Witten index (2.42) for the theory is 3 and there are 3 irreducible flat connections given by the following Bethe equation:

$$
\exp \left(\partial_{Z} S_{0}\right)=1 \quad \Rightarrow \quad \frac{z-1}{z^{3}}=1 .
$$

The solutions and the corresponding classical actions are

$$
\begin{array}{rll}
\mathcal{A}^{\overline{\text { geom }}}: Z=0.1406+0.703858 i & \left(\ell_{z}=0\right), & S_{0}=1.1852-0.942707 i, \\
\mathcal{A}^{\text {geom }}: Z=0.1406-0.703858 i & \left(\ell_{z}=0\right), & S_{0}=1.1852+0.942707 i, \\
\mathcal{A}^{3 \mathrm{rd}}: Z=0.2812+\pi i & \left(\ell_{z}=1\right), & S_{0}=-10.5951 .
\end{array}
$$


The manifold has vanishing $H_{1}\left(M_{3}, \mathbb{Z}_{2}\right)$ and there are no $\mathbb{Z}_{2}$ flat connections. The perturbative invariants $\left\{S_{n}\right\}$ up to $n=5$ for the irreducible flat connections are

$$
\begin{aligned}
S_{0}(\text { Weeks; } N=2)= & \operatorname{Li}_{2}\left(e^{-Z}\right)+2 \pi i \ell_{z} Z-Z^{2}, \\
S_{1}(\text { Weeks; } N=2)= & -\frac{1}{2} \log \left(1-\frac{3}{2 z}\right) \\
S_{2}(\text { Weeks; } N=2)= & \frac{46 z^{2}-72 z+27}{24(2 z-3)^{3}}, \\
S_{3}(\text { Weeks; } N=2)= & \frac{z\left(5 z^{4}+9 z^{3}-41 z^{2}+36 z-9\right)}{2(3-2 z)^{6}}, \\
S_{4}(\text { Weeks; } N=2)= & \frac{(z-1) z\left(988 z^{6}+45700 z^{5}-9642 z^{4}-149328 z^{3}+140103 z^{2}\right)}{720(2 z-3)^{9}}, \\
& +\frac{(z-1) z(-18225 z-9477)}{720(2 z-3)^{9}}, \\
S_{5}(\text { Weeks; } N=2)= & \frac{(1-z) z\left(4 z^{9}-4022 z^{8}-34158 z^{7}+64404 z^{6}+74400 z^{5}-209358 z^{4}\right)}{24(3-2 z)^{12}} \\
& +\frac{(1-z) z\left(121041 z^{3}+1296 z^{2}-15795 z+2187\right)}{24(3-2 z)^{12}} .
\end{aligned}
$$

Using the perturbative invariants, one can check that

$$
\begin{aligned}
& \mathcal{I}_{\text {top }}\left(q ; \mathcal{T}_{N=2}\left[M_{3}=\text { Weeks }\right]\right) \\
& =\left.\frac{1}{2} \sum_{\frac{z-1}{z^{3}}=1} \exp \left(2 S_{1}\left(M_{3} ; N\right)+2 S_{3}\left(M_{3} ; N\right) \hbar^{2}+2 S_{5}\left(M_{3} ; N\right) \hbar^{4}+\mathcal{O}\left(\hbar^{6}\right)\right)\right|_{M_{3}=\text { Weeks }} ^{N=2} \\
& =0+\mathcal{O}\left(\hbar^{6}\right) .
\end{aligned}
$$

Again, the refined index vanishes in a highly non-trivial way — we have checked the vanishing up to $\mathcal{O}\left(\hbar^{10}\right)$.

Example: $M_{3}=\left(S^{3} \backslash 5_{1}^{2}\right)_{P_{1} / Q_{1}=-6, P_{2} / Q_{2}=-3 / 2}$. This manifold is the third smallest orientable closed hyperbolic 3 -manifold [58], and its volume is

$$
\operatorname{vol}\left(M_{3}\right) \simeq 1.014942
$$

The corresponding $3 \mathrm{~d}$ gauge theory for $N=2$ is [34]

$$
\mathcal{T}_{N=2}\left[M_{3}\right]=\left(\mathrm{U}(1)_{k=-3 / 2} \text { coupled to a single chiral } \Phi \text { of charge }+1\right) .
$$

The classical Chern-Simons action is

$$
S_{0}=\operatorname{Li}_{2}\left(e^{-Z}\right)+2 \pi i \ell_{z} Z-\frac{1}{2} Z^{2} .
$$

The Witten index of (2.52) is 2 , and the two solutions to the Bethe equations $\exp \left(\partial_{Z} S_{0}\right)=1$ correspond to the equivalence classes of the two geometric flat connections (in this case $\left.H_{1}\left(M_{3}, \mathbb{Z}_{2}\right)=\mathbb{Z}_{2}\right)$. Using the state-integral model for the 3 -manifold, we have checked that the refined index computed using the $3 \mathrm{~d}-3 \mathrm{~d}$ relation (2.29) vanishes up to $\mathcal{O}\left(\hbar^{10}\right)$. 


\subsubsection{Integer expansion of the twisted index}

On a generic Riemann surface $\Sigma_{g}$, thus with no $q$-refinement, we expect

$$
\mathcal{I}_{\Sigma_{g}}\left(\mathcal{T}_{N=2}\left[M_{3}\right]\right) \in \mathbb{Z}
$$

According to the $3 \mathrm{~d}-3 \mathrm{~d}$ relation (2.35), the (unrefined) twisted index is determined by the one-loop invariants $S_{1}^{\alpha}\left(M_{3} ; N\right)$. The one-loop part is simply related to the mathematical quantity called analytic Ray-Singer torsion, see (2.13). Computing such topological quantity using its definition (2.14) is quite a challenging task, since we need to know the full spectrum of the Laplacians on the 3-manifold. There are two simpler alternative ways to compute the Ray-Singer torsion: i) using a state-integral model for complex Chern-Simons theory, and ii) using Cheeger-Muller theorem. See appendix A for a brief introduction to the state-integral model. Cheeger-Muller theorem claims equivalence between the analytic Ray-Singer torsion and the combinatorial Reidermeister torsion. The combinatorial torsion can be computed from so-called Fox calculus on the $\operatorname{SL}(N, \mathbb{C})$ representations of $\pi_{1}\left(M_{3}\right)$. In [51] it is explicitly checked that the two simpler approaches give the same answer in a large number of examples. In this subsection, we give the explicit expression for the combinatorial Reidermeister torsion $\operatorname{Tor}\left[\mathcal{A}^{\alpha} ; M_{3}\right]$ (equal to the analytic Ray-Singer torsion) twisted by $\operatorname{SL}(2, \mathbb{C})$ irreducible flat connections $\mathcal{A}^{\alpha}$ on $M_{3}=\left(S^{3} \backslash \mathbf{4}_{1}\right)_{P / Q}$ and check the integrality of $\mathcal{I}_{\Sigma_{g}}\left(\mathcal{T}_{N=2}\left[M_{3}\right]\right)$ in (2.35).

Example: $\left(\boldsymbol{S}^{\mathbf{3}} \backslash \mathbf{4}_{\mathbf{1}}\right)_{P / Q}$. These manifolds are hyperbolic for all $P / Q \in \mathbb{Q} \cup\{\infty\}$ but the following 10 exceptional slopes:

$$
\text { Exceptional slopes of } S^{3} \backslash \mathbf{4}_{1}: P / Q=\{0, \pm 1, \pm 2, \pm 3, \pm 4, \infty\} \text {. }
$$

The fundamental groups of these 3-manifolds are

$$
\begin{aligned}
& \pi_{1}\left(S^{3} \backslash \mathbf{4}_{1}\right)=\left\langle a, b: a b^{-1} a^{-1} b a=b a b^{-1} a^{-1} b\right\rangle, \\
& \pi_{1}\left(M_{3}\right)=\left\langle a, b: a b^{-1} a^{-1} b a=b a b^{-1} a^{-1} b, \mathbf{m}^{P} \mathbf{l}^{Q}=1\right\rangle, \text { where } \\
& \mathbf{m}:=a, \quad \mathbf{l}:=a b^{-1} a b a^{-2} b a b^{-1} a^{-1} .
\end{aligned}
$$

The set $\chi_{\text {irred }}\left(M_{3}=\left(S^{3} \backslash \mathbf{4}_{1}\right)_{P / Q} ; N=2\right)$ can be obtained by solving the following matrix equations:

$$
\chi_{\text {irred }}\left(M_{3} ; N=2\right)=\left\{A=\left(\begin{array}{cc}
m & 1 \\
0 & m^{-1}
\end{array}\right), B=\left(\begin{array}{cc}
m & 0 \\
y & m^{-1}
\end{array}\right): \text { matrix eqs. }\right\} / \mathbb{Z}_{2},
$$

where the matrix equations are

$$
\begin{aligned}
& A B^{-1} A^{-1} B A=B A B^{-1} A^{-1} B \quad \text { and } \quad M^{P} L^{Q}=\mathbb{I} \quad \text { with } \\
& M:=A, \quad L=A B^{-1} A B A^{-2} B A B^{-1} A^{-1} .
\end{aligned}
$$

Indeed, one can show ${ }^{5}$ that $a$ and $b$ are related to each other by a conjugation in $\pi_{1}\left(M_{3}\right)$, and so their corresponding holonomy matrices $A$ and $B$ are related by a conjugation in

\footnotetext{
${ }^{5}$ From the first line of $(2.56)$ it follows $b=\left(a^{-1} b a b^{-1}\right) a\left(b a^{-1} b^{-1} a\right)$.
} 
$\mathrm{SL}(2, \mathbb{C})$. Using the $\mathrm{SL}(2, \mathbb{C})$ conjugation, we fix the matrices $A$ and $B$ as above. There is a residual $\mathbb{Z}_{2}$ gauge symmetry acting as

$$
\mathbb{Z}_{2}:(m, y) \leftrightarrow\left(m^{-1}, y\right) .
$$

The matrix equations give algebraic equations for $m$ and $y$, which can be solved numerically. When $P / Q=5$, we have the following 4 solutions which can be identified with the four Bethe vacua in (2.43):

$$
\begin{array}{ll}
(m, y)=(1.20331+0.780836 i, 0.992448+0.513116 i) & \leftrightarrow \mathcal{A}^{\overline{g e o m}} \\
(m, y)=(1.20331-0.780836 i, 0.992448-0.513116 i) & \leftrightarrow \mathcal{A}^{\text {geom }} \\
(m, y)=(0.164478+0.986381 i, 3.49022) & \leftrightarrow \mathcal{A}^{3 \mathrm{rd}} \\
(m, y)=(-0.452583+0.891722 i, 2.52489) & \leftrightarrow \mathcal{A}^{4 \mathrm{th}} .
\end{array}
$$

The torsion can be computed from the following formula (see the appendix of [33] for the derivation):

$$
\begin{aligned}
& \left.\exp \left(-2 S_{1}^{\alpha}\left(M_{3} ; N=2\right)\right)\right|_{M_{3}=\left(S^{3} \backslash \mathbf{4}_{1}\right)_{P / Q}} \\
& =\left(P\left(\ell-\frac{1}{\ell}\right) \frac{m^{4}}{\left(m^{4}-1\right)\left(4-2 m^{2}+4 m^{4}\right)}+Q\right) \frac{2 m^{2}+\frac{2}{m^{2}}-1}{\left(1-m^{2 R} \ell^{2 S}\right)\left(1-m^{-2 R} \ell^{-2 S}\right)} .
\end{aligned}
$$

Here $\ell$ is defined from the following relation:

$$
L=\left(\begin{array}{cc}
\ell & * \\
0 & \ell^{-1}
\end{array}\right) .
$$

Indeed, after solving the matrix equations, the two $\mathrm{SL}(2, \mathbb{C})$ matrices $M$ and $L$ commute since $\mathbf{m l}=\mathbf{l} \mathbf{m}$ in $\pi_{1}\left(S^{3} \backslash \mathbf{4}_{1}\right)$ and in $\pi_{1}\left(M_{3}\right)$, as one can show. ${ }^{6}$ So, after solving the matrix equations, $L$ takes the upper-triangular form as above. One can show that the constraint of $\pi_{1}\left(S^{3} \backslash \mathbf{4}_{1}\right)$ implies

$$
2+\ell+\ell^{-1}-m^{4}+m^{2}+m^{-2}-m^{-4}=0
$$

which is the A-polynomial of $\mathbf{4}_{1}$. Then, $(R, S)$ are coprime integers chosen such that $P S-$ $Q R=1$. For given $(P, Q)$, the choice is not unique but the above torsion is independent of the choice since the matrix equation $M^{P} L^{Q}=\mathbb{I}$ implies $m^{P} \ell^{Q}=1$. By applying the formula to $P / Q=5$, we find that

$$
\begin{aligned}
& \left.\exp \left(-2 S_{1}^{\alpha}\left(M_{3} ; N=2\right)\right)\right|_{M_{3}=\left(S^{3} \backslash \mathbf{4}_{1}\right)_{P / Q=5}} ^{\alpha=\overline{\text { eeom }}}=1.90538-0.568995 i \\
& \left.\exp \left(-2 S_{1}^{\alpha}\left(M_{3} ; N=2\right)\right)\right|_{M_{3}=\left(S^{3} \backslash \mathbf{4}_{1}\right)_{P / Q=5}} ^{\alpha=\text { egom }}=1.90538+0.568995 i \\
& \left.\exp \left(-2 S_{1}^{\alpha}\left(M_{3} ; N=2\right)\right)\right|_{M_{3}=\left(S^{3} \backslash \mathbf{4}_{1}\right)_{P / Q=5}} ^{\alpha=\operatorname{ld}}=-2.57085 \\
& \left.\exp \left(-2 S_{1}^{\alpha}\left(M_{3} ; N=2\right)\right)\right|_{M_{3}=\left(S^{3} \backslash \mathbf{4}_{1}\right)_{P / Q=5}} ^{\alpha=4 \mathrm{th}}=-1.73992 .
\end{aligned}
$$

\footnotetext{
${ }^{6}$ From the first line of (2.56) follow $a^{2} b^{-1} a b a^{-1}=a b^{-1} a b a^{-1} b$ and $b^{-1} a^{-1} b a b^{-1}=a^{-1} b a b^{-1} a^{-1}$.
} 
One can check that the numerical values are equal to $e^{-2 S_{1}^{\alpha}}$ in $(2.40)$ for the Bethe vacua (2.43) computed from the state-integral model.

Combining the torsion computations in (2.57) and (2.61) with the $3 \mathrm{~d}-3 \mathrm{~d}$ relation in (2.35), we obtain the following concrete expression for the twisted index:

$$
\begin{aligned}
\mathcal{I}_{\Sigma_{g}}\left(\mathcal{T}_{N=2}\left[M_{3}\right]\right)= & \frac{1}{\left|\operatorname{Hom}\left[\pi_{1}\left(M_{3}\right) \rightarrow \mathbb{Z}_{2}\right]\right|} \\
& \times \sum_{(m, y) \in \chi_{\text {irred }} \text { in }(2.57)}\left(\frac{2}{\left|\operatorname{Hom}\left[\pi_{1}\left(M_{3}\right) \rightarrow \mathbb{Z}_{2}\right]\right|}\left(\exp \left(-2 S_{1}\right) \text { in }(2.61)\right)\right)^{g-1}
\end{aligned}
$$

for $M_{3}=\left(S^{3} \backslash \mathbf{4}_{1}\right)_{P / Q}$. Note that

$$
\left|\operatorname{Hom}\left[\pi_{1}\left(M_{3}\right) \rightarrow \mathbb{Z}_{2}\right]\right|= \begin{cases}1 & \text { for odd } P \\ 2 & \text { for even } P .\end{cases}
$$

Using the expression above, one can check that $\mathcal{I}_{\Sigma_{g}}\left(\mathcal{I}_{N=2}\left[M_{3}\right]\right) \in \mathbb{Z}$, which is quite non-trivial.

For example, using the torsions in (2.64) for the case $P / Q=5$, one obtains

$$
\begin{aligned}
& \left.\mathcal{I}_{\Sigma_{g}}\left(\mathcal{T}_{N=2}\left[M_{3}\right]\right)\right|_{M_{3}=\left(S^{3} \backslash \mathbf{4}_{1}\right)_{P / Q=5}} \\
& =\left.\sum_{\alpha} 2^{g-1} \exp \left(-2(g-1) S_{1}^{\alpha}\left(M_{3} ; N=2\right)\right)\right|_{M_{3}=\left(S^{3} \backslash \mathbf{4}_{\mathbf{1}}\right)_{P / Q=5}} \\
& =\left\{0_{g=0}, 4_{g=1},-1_{g=2}, 65_{g=3},-97_{g=4}, \ldots\right\} .
\end{aligned}
$$

In fact, we can give a precise algebraic characterization of these numbers. The Bethe vacua (2.43) are the four solutions to the algebraic equation $z^{4}+z-1=0$. From (2.40), the Ray-Singer torsion contribution from each irreducible flat connection is

$$
2 e^{-2 S_{1}}=\frac{3 z-4}{z^{2}} \equiv w .
$$

One can easily show that the Bethe ansatz algebraic equation implies

$$
w^{4}+w^{3}-32 w^{2}+283=0 .
$$

In other words, the four values of $w$ are the roots of an algebraic equation with integer coefficients. This implies that $\sum_{i=1}^{4} w_{i}^{g-1} \in \mathbb{Z}$ for all $g \geq 1$, and it allows to determine the integers values of the index in (2.67) in an algebraic way from the coefficients of the equation. Since the equation has no linear term, it also implies (for $g=0$ ) that $\sum_{i=1}^{4} w_{i}^{-1}=0$ which is our vanishing conjecture.

The case of Weeks' manifold can be treated in a similar way. From (2.49), the Bethe ansatz equation - or equation for the irreducible flat connections - is $z^{3}-z+1=0$. The contributions from Ray-Singer torsion are $2 e^{-2 S_{1}}=(2 z-3) / z \equiv w$, and one easily shows that

$$
w^{3}-3 w^{2}-23=0 .
$$


The fact that $w$ 's satisfy an algebraic equation with integer coefficients and no linear term implies that $\sum_{i=1}^{3} w_{i}^{g-1} \in \mathbb{Z}$ for $g \geq 1$, and that it vanishes for $g=0$.

In the case of $\left(S^{3} \backslash \mathbf{4}_{1}\right)_{P / Q}$ with $P / Q=6$, there are 6 irreducible flat connections in $\chi_{\text {irred }}\left(M_{3} ; N=2\right)$ but there are only 3 equivalence classes $\left[\mathcal{A}^{\alpha}\right] \in \frac{\chi_{\text {irred }}}{\operatorname{Hom}\left[\pi_{1}\left(M_{3}\right) \rightarrow \mathbb{Z}_{2}\right]}$. The numerical values of the torsion for the three classes are

$$
\begin{aligned}
\left\{\exp \left(-2 S_{1}^{\alpha}\left(M_{3} ; N=2\right)\right)\right\}_{\left[\mathcal{A}^{\alpha}\right] \in \frac{\chi_{\text {irred }}}{\operatorname{Hom}\left[\pi_{1}\left(M_{3}\right) \rightarrow \mathbb{Z}_{2}\right]}} \quad \text { for } M_{3}=\left(S^{3} \backslash \mathbf{4}_{1}\right)_{P / Q=6} \\
=\{4.30835-1.99637 i, 4.30835+1.99637 i,-2.61671\} .
\end{aligned}
$$

Then, we see that

$$
\begin{aligned}
& \left.\mathcal{I}_{\Sigma_{g}}\left(\mathcal{T}_{N=2}\left[M_{3}\right]\right)\right|_{M_{3}=\left(S^{3} \backslash \mathbf{4}_{1}\right)_{P / Q=6}} \\
& \left.\sum_{\left[\mathcal{A}^{\alpha}\right] \in \in_{\text {irred }}^{\operatorname{Hom}\left[\pi_{1}\left(M_{3}\right) \rightarrow \mathbb{Z}_{2}\right]}} \exp \left(-2(g-1) S_{1}^{\alpha}\left(M_{3} ; N=2\right)\right)\right|_{M_{3}=\left(S^{3} \backslash \mathbf{4}_{\mathbf{1}}\right)_{P / Q=6}} \\
& =\left\{0_{g=0}, 3_{g=1}, 6_{g=2}, 36_{g=3}, 39_{g=4}, \ldots\right\} .
\end{aligned}
$$

This integrality constitutes a non-trivial consistency check for all the subtle factors in the $3 \mathrm{~d}-3 \mathrm{~d}$ relation (2.35). These factors will play an important role in the computation of the $\log N$ subleading corrections to the twisted indices at large $N$.

\section{Large $N$ limit of the indices}

We consider now the large $N$ limit of the three types of indices of $\mathcal{T}_{N}\left[M_{3}\right]$ using the $3 \mathrm{~d}-3 \mathrm{~d}$ relations in $(2.27),(2.29)$ and $(2.35)$. Through the $3 \mathrm{~d}-3 \mathrm{~d}$ relations, all indices are related to the holomorphic blocks $(2.10)$ of $\operatorname{SL}(N, \mathbb{C})$ Chern-Simons theory on $M_{3}$. The blocks can be perturbatively expanded in the holomorphic coupling. We have two expansion parameters, $1 / N$ and $\hbar$. We first consider the perturbative expansion in $\hbar$, and then take the large $N$ limit of the perturbative expansion coefficients $\left\{S_{n}\left(M_{3} ; N\right)\right\}$.

\subsection{Perturbative Chern-Simons invariants}

There are two canonical $\operatorname{SL}(N, \mathbb{C})$ irreducible flat connections on any hyperbolic 3-manifold $M_{3}$, denoted $\mathcal{A}_{N}^{\text {geom }}$ and $\mathcal{A}_{N}^{\overline{\text { geom }}}$ :

$$
\mathcal{A}_{N}^{\text {geom }}=\rho_{N}(\omega+i e), \quad \mathcal{A}_{N}^{\overline{\text { geom }}}=\rho_{N}(\omega-i e) .
$$

According to Mostow's rigidity theorem [59], there is a unique hyperbolic metric on $M_{3}$ satisfying $R_{\mu \nu}=-2 g_{\mu \nu}$. Here $e$ and $\omega$ are dreibein and spin connection of the hyperbolic structure, respectively. Both of them can be considered as $\mathfrak{s o}(3)$-valued 1-forms and they form two $\mathrm{SL}(2, \mathbb{C})$ flat connections, $\omega \pm i e$. On the other hand, $\rho_{N}: \mathrm{SL}(2) \rightarrow \mathrm{SL}(N)$ is the principal embedding. A characteristic property of the two irreducible flat connections is

$$
\operatorname{Im}\left[S_{0}^{\overline{\text { geom }}}\right] \leq \operatorname{Im}\left[S_{0}^{\alpha}\right] \leq \operatorname{Im}\left[S_{0}^{\text {geom }}\right] \quad \forall\left[\mathcal{A}^{\alpha}\right] \in \frac{\chi_{\text {irred }}\left(M_{3} ; N\right)}{\operatorname{Hom}\left[\pi_{1}\left(M_{3}\right) \rightarrow \mathbb{Z}_{N}\right]}
$$


The equality holds for and only for $\alpha=$ geom or $\alpha=\overline{\text { geom}}$. The classical and one-loop parts around the flat connections can be expressed in terms of the hyperbolic volume of the 3-manifold, $\operatorname{vol}\left(M_{3}\right)$, and the complex length spectrum $\left\{\ell_{\mathbb{C}}(\gamma)\right\}$ :

$$
\begin{aligned}
& \operatorname{Im}\left[S_{0}^{\text {geom }}\left(M_{3} ; N\right)\right]=\frac{\left(N^{3}-N\right)}{6} \operatorname{vol}\left(M_{3}\right) \\
& \operatorname{Re}\left[S_{1}^{\text {geom }}\left(M_{3} ; N\right)\right]=-\frac{\operatorname{vol}\left(M_{3}\right)}{12 \pi}\left(2 N^{3}-N-1\right)-\frac{1}{2} \sum_{\gamma} \sum_{m=1}^{N-1} \sum_{k=m+1}^{\infty} \log \left|1-e^{-k \ell_{\mathbb{C}}(\gamma)}\right| .
\end{aligned}
$$

Here we defined

$$
\operatorname{vol}\left(M_{3}\right):=\left(\text { volume of } M_{3} \text { measured in the unique hyperbolic metric) } .\right.
$$

Moreover $\sum_{\gamma}$ is the sum over the non-trivial primitive geodesics $\gamma \in M_{3}$, and $\ell_{\mathbb{C}}(\gamma)$ is the complexified geodesic length of $\gamma$, which is defined by the following relation:

$$
\operatorname{Tr} \operatorname{Pexp}\left(-\oint_{\gamma} \mathcal{A}_{N=2}^{\text {geom }}\right)=e^{\frac{1}{2} \ell_{\mathbb{C}}(\gamma)}+e^{-\frac{1}{2} \ell_{\mathbb{C}}(\gamma)}, \quad \operatorname{Re}\left[\ell_{\mathbb{C}}\right]>0 .
$$

The real part of $\ell_{\mathbb{C}}$ measures the geodesic length.

Let us explain the origin of the two formulas in (3.3). The classical part of the ChernSimons action can be obtained using the following computation $\left(h_{1}\right.$ and $h_{2}$ are non-zero elements in $\mathfrak{s l}(2, \mathbb{C}))$ :

$$
\begin{aligned}
& \operatorname{Im}\left[S_{0}^{\text {geom }}\left(M_{3} ; N\right)\right]=\operatorname{Im}\left[-2 \pi \operatorname{CS}\left[\mathcal{A}_{N}^{\text {geom }}, M_{3}\right]\right] \\
& =\frac{\operatorname{Tr}\left(\rho_{N}\left(h_{1}\right) \rho_{N}\left(h_{2}\right)\right)}{\operatorname{Tr}\left(\rho_{N=2}\left(h_{1}\right) \rho_{N=2}\left(h_{2}\right)\right)} \operatorname{Im}\left[-2 \pi \operatorname{CS}\left[\mathcal{A}_{N=2}^{\text {geom }}, M_{3}\right]\right] \\
& =\frac{\left(N^{3}-N\right)}{6} \operatorname{Im}\left[-2 \pi \operatorname{CS}\left[\mathcal{A}=\omega+i e, M_{3}\right]\right]=-\left.\frac{\left(N^{3}-N\right)}{24} \int_{M_{3}} \sqrt{g}(R+2)\right|_{R_{\mu \nu}=-2 g_{\mu \nu}} \\
& =\frac{\left(N^{3}-N\right)}{6} \int_{M_{3}} \sqrt{g}=\frac{\left(N^{3}-N\right)}{6} \operatorname{vol}\left(M_{3}\right)
\end{aligned}
$$

The expression for the one-loop part $S_{1}^{\text {geom }}$ is derived in [33] from results $[60,61]$ in mathematics using Selberg's trace formula. To see the $1 / N$ expansion more explicitly, we can write

$$
\begin{aligned}
& \sum_{\gamma} \sum_{m=1}^{N-1} \sum_{k=m+1}^{\infty} \log \left|1-e^{-k \ell_{\mathbb{C}}(\gamma)}\right| \\
& =-\operatorname{Re} \sum_{\gamma} \sum_{s=1}^{\infty} \frac{1}{s}\left(\frac{e^{-s \ell_{\mathbb{C}}}}{1-e^{-s \ell_{\mathbb{C}}}}\right)^{2}+\operatorname{Re} \sum_{\gamma} \sum_{s=1}^{\infty} \frac{1}{s}\left(\frac{e^{-s(N+1) \ell_{\mathbb{C}} / 2}}{1-e^{-s \ell_{\mathbb{C}}}}\right)^{2} .
\end{aligned}
$$

The first term is independent of $N$ while the second one is exponentially suppressed at large $N$. 
The higher perturbative invariants $S_{n}^{\text {geom }}(n \geq 2)$ are conjectured to behave at large $N$ as $[62]^{7}$

$$
\begin{aligned}
\operatorname{Im}\left[S_{2}^{\text {geom }}\left(M_{3} ; N\right)\right] & =-\frac{N^{3}}{24 \pi^{2}} \operatorname{vol}\left(M_{3}\right)+\text { subleading, } \\
\lim _{N \rightarrow \infty} \frac{1}{N^{3}} \operatorname{Re}\left[S_{2 n+1}^{\text {geom }}\left(M_{3} ; N\right)\right] & =0 \quad(n \geq 1), \\
\lim _{N \rightarrow \infty} \frac{1}{N^{3}} \operatorname{Im}\left[S_{2 n}^{\text {geom }}\left(M_{3} ; N\right)\right] & =0 \quad(n \geq 2) .
\end{aligned}
$$

Unlike $S_{0}^{\text {geom }}$ and $S_{1}^{\text {geom }}$, we have conjectures only for the leading $\mathcal{O}\left(N^{3}\right)$ terms at large $N$. The large $N$ behavior of $S^{\overline{\mathrm{geom}}}$ can be obtained using the following general fact:

$$
S_{n}^{\bar{\alpha}}=\left(S_{n}^{\alpha}\right)^{*} .
$$

The conjecture (3.8) has been numerically tested in many examples up to seven loops. One way to understand the conjecture is the S-duality of complex Chern-Simons theory [63] relating $\hbar \leftrightarrow-\frac{4 \pi^{2}}{\hbar}$. At finite $N$, the S-duality is only seen after Borel-Padé resummation [64]. At large $N$, on the other hand, the perturbative expansion becomes a convergent series as far as the leading $\mathcal{O}\left(N^{3}\right)$ behavior is concerned, and one can see the S-duality directly in the $\mathcal{O}\left(N^{3}\right)$ coefficients of the perturbative invariants.

\subsection{Superconformal index}

\subsubsection{Leading $N^{3}$ behavior}

Let us set $q:=e^{-\omega}$ with $\operatorname{Im} \omega<0$ and consider an expansion for $|\omega| \ll 1$. Combining the 3d-3d relation (2.27) with the large $N$ behavior (3.3) and (3.8), we find

$$
\begin{aligned}
& \mathcal{I}_{\text {sci }}\left(q ; \mathcal{T}_{N}\left[M_{3}\right]\right)=\frac{\left|\operatorname{Hom}\left[\pi_{1}\left(M_{3}\right) \rightarrow \mathbb{Z}_{N}\right]\right|}{N} \sum_{\left[\mathcal{A}^{\alpha}\right] \in \frac{\chi_{\text {irred }}\left(M_{3} ; N\right)}{\operatorname{Hom}\left[\pi_{1}\left(M_{3}\right) \rightarrow \mathbb{Z}_{N}\right]}} B^{\alpha}(q) B^{\bar{\alpha}}\left(q^{-1}\right) \\
& \stackrel{N \rightarrow \infty}{\longrightarrow} \frac{\left|\operatorname{Hom}\left[\pi_{1}\left(M_{3}\right) \rightarrow \mathbb{Z}_{N}\right]\right|}{N} B^{\text {geom }}(q) B^{\overline{\operatorname{geom}}}\left(q^{-1}\right)+\ldots \\
& =\frac{\left|\operatorname{Hom}\left[\pi_{1}\left(M_{3}\right) \rightarrow \mathbb{Z}_{N}\right]\right|}{N} \exp \left(\sum_{n=0}^{\infty}(-\omega)^{n-1} S_{n}^{\text {geom }}\right) \exp \left(\sum_{n=0}^{\infty} \omega^{n-1}\left(S_{n}^{\text {geom }}\right)^{*}\right)+\ldots \\
& =\frac{\left|\operatorname{Hom}\left[\pi_{1}\left(M_{3}\right) \rightarrow \mathbb{Z}_{N}\right]\right|}{N} \exp \left\{\left(-\frac{i N^{3}}{3 \omega}-\frac{N^{3}}{3 \pi}+\frac{i \omega N^{3}}{12 \pi^{2}}\right) \operatorname{vol}\left(M_{3}\right)+\text { subleading in } \frac{1}{N}\right\}+\ldots
\end{aligned}
$$

where dots stand for exponentially smaller terms in the $1 / N$ expansion. In particular, because of (3.2), all irreducible flat connections $\mathcal{A}^{\alpha}$ other than $\alpha=$ geom give an exponentially suppressed contribution to the second line. We thus find

$$
\begin{aligned}
\log \mathcal{I}_{\text {sci }}\left(q ; \mathcal{T}_{N}\left[M_{3}\right]\right) & =\frac{i N^{3}}{12 \pi^{2}} \operatorname{vol}\left(M_{3}\right) \frac{(\omega+2 \pi i)^{2}}{\omega}+(\text { subleading in } 1 / N) \\
& =\frac{i L^{2}}{8 G_{(4)}} \frac{(\omega+2 \pi i)^{2}}{\omega}+\left(\text { subleading in } G_{(4)} / L^{2}\right) .
\end{aligned}
$$

\footnotetext{
${ }^{7}$ In [62], $\mathcal{A}^{\overline{\text { geom }}}$ is denoted as $\mathcal{A}^{\text {conj }}$.
} 


\begin{tabular}{|c|c|c|}
\hline & $\operatorname{SL}(N, \mathbb{C})$ CS theory on $M_{3}$ & Supergravity on $\operatorname{AdS}_{4} \times M_{3} \times \widetilde{S}^{4}$ \\
\hline \multirow{3}{*}{$\log N$} & Quotient by & Two-form zero-modes on $\operatorname{AdS}_{4}$ from \\
& residual gauge symmetry: & 2-form ghost field \\
& $\frac{1}{\left|\operatorname{Stab}\left(\mathcal{A}^{\text {geom }}\right)\right|}=\frac{1}{N}$ & in 11d supergravity \\
\hline \multirow{3}{*}{$b_{1}\left(M_{3}\right) \log N$} & Degeneracy of $\left\{\mathcal{A}^{\eta \otimes \alpha}\right\}$ from & Two-form zero-modes on $\mathrm{AdS}_{4}$ \\
& tensoring with $\mathbb{Z}_{N}$ flat connections: & from 3-form potential $C_{(3)}$ \\
& $\left|\operatorname{Hom}\left[\pi_{1}\left(M_{3}\right) \rightarrow \mathbb{Z}^{N}\right]\right| \sim N^{b_{1}\left(M_{3}\right)}$ & in 11d supergravity \\
\hline
\end{tabular}

Table 1. Comparison between the origin of logarithmic corrections to the superconformal index of $\mathcal{T}_{N}\left[M_{3}\right]$, from the $3 \mathrm{~d}-3 \mathrm{~d}$ dual $\mathrm{SL}(N, \mathbb{C})$ Chern-Simons theory and from the holographic supergravity.

Here $L$ is the radius of $\mathrm{AdS}_{4}$ and $G_{(4)}$ is the $4 \mathrm{~d}$ Newton constant. We used the relation [33]

$$
\frac{L^{2}}{G_{(4)}}=\frac{2 N^{3}}{3 \pi^{2}} \operatorname{vol}\left(M_{3}\right) \text {. }
$$

As we will show, this large $N$ free energy nicely matches with the entropy function in (4.18).

\subsubsection{Logarithmic subleading corrections}

The large $N$ logarithmic subleading correction - proportional to $\log N$ - is independent of the continuous deformation parameter $\omega$. So we can compute it in the $\omega \rightarrow 0$ limit, which is controlled by the overall factor $\left|\operatorname{Hom}\left[\pi_{1}\left(M_{3}\right) \rightarrow \mathbb{Z}_{N}\right]\right| / N$ and the perturbative expansion of $S_{0}^{\text {geom }}$ and $S_{1}^{\text {geom }}$. We have

$$
\begin{aligned}
& \left.\log \mathcal{I}_{\text {sci }}\left(q ; \mathcal{T}_{N}\left[M_{3}\right]\right)\right|_{\omega \rightarrow 0, N \gg 1} \\
& =\frac{2}{i \omega} \operatorname{Im}\left[S_{0}^{\text {geom }}\left(M_{3} ; N\right)\right]+2 \operatorname{Re}\left[S_{1}^{\text {geom }}\left(M_{3} ; N\right)\right]+\log \frac{\left|\operatorname{Hom}\left[\pi_{1}\left(M_{3}\right) \rightarrow \mathbb{Z}_{N}\right]\right|}{N}+\mathcal{O}(\omega) .
\end{aligned}
$$

From (3.3) and (3.7) we see that there is no $\log N$ corrections from the first two terms, $\operatorname{Im}\left[S_{0}^{\text {geom }}\right]$ and $\operatorname{Re}\left[S_{1}^{\text {geom }}\right]$. The $\log N$ correction only comes from the last term, namely

$$
\log \frac{\left|\operatorname{Hom}\left[\pi_{1}\left(M_{3}\right) \rightarrow \mathbb{Z}_{N}\right]\right|}{N}=\log \frac{\left|\operatorname{Hom}\left[H_{1}\left(M_{3}, \mathbb{Z}\right) \rightarrow \mathbb{Z}_{N}\right]\right|}{N}=\left(b_{1}\left(M_{3}\right)-1\right) \log N .
$$

This matches the supergravity analysis in (5.5) with $g=0$. The comparison between the computation of logarithmic corrections in $\operatorname{SL}(N, \mathbb{C})$ Chern-Simons theory and in supergravity is summarized in table 1.

\subsection{Twisted indices for $g>1$}

A remarkable aspect of the $3 \mathrm{~d}-3 \mathrm{~d}$ relation for twisted indices (2.35) is that only the one-loop invariant $S_{1}^{\alpha}$ appears in the relation. Since we know the full perturbative $1 / N$ expansion of the one-loop part, we can compute the full perturbative $1 / N$ corrections to the twisted index of $\mathcal{T}_{N}\left[M_{3}\right]$ theory. Using this idea, the full perturbative $1 / N$ corrections were studied in [33]. In that work, the $3 \mathrm{~d}-3 \mathrm{~d}$ relation (2.31) was limited to 3-manifolds with vanishing 
$H_{1}\left(M_{3}, \mathbb{Z}_{N}\right)$. With the more general $3 \mathrm{~d}$-3d relation for twisted indeces $(2.35)$, we can repeat the large $N$ analysis for general closed hyperbolic 3-manifolds $M_{3}$. Interestingly, the logarithmic subleading correction $\log N$ can detect the first Betti number $b_{1}\left(M_{3}\right)$ of the 3 -manifold. See [65] for previous studies on full perturbative $1 / N$ corrections to the twisted index of other classes of $3 \mathrm{~d}$ theories.

\subsubsection{All orders in $1 / N$}

Combining the $3 \mathrm{~d}-3 \mathrm{~d}$ relation (2.35) with the large $N$ behavior (3.3), we obtain for $g>1$ :

$$
\begin{aligned}
& \mathcal{I}_{\Sigma_{g}}\left(\mathcal{T}_{N}\left[M_{3}\right]\right) \\
& \stackrel{N \rightarrow \infty}{\longrightarrow} \frac{N^{g-1}}{\left|\operatorname{Hom}\left[\pi_{1}\left(M_{3}\right) \rightarrow \mathbb{Z}_{N}\right]\right|^{g-1}}\left(e^{-2(g-1) S_{1}^{\text {geom }}\left(M_{3} ; N\right)}+\text { c.c. }+\ldots\right) \\
& =\exp \left\{(g-1) \frac{\operatorname{vol}\left(M_{3}\right)}{6 \pi}\left(2 N^{3}-N-1\right)-(g-1) \operatorname{Re} \sum_{\gamma} \sum_{s=1}^{\infty} \frac{1}{s}\left(\frac{e^{-s \ell_{\mathbb{C}}}}{1-e^{-s \ell_{\mathbb{C}}}}\right)^{2}\right. \\
& \left.-(g-1) \log \frac{\left|\operatorname{Hom}\left[\pi_{1}\left(M_{3}\right) \rightarrow \mathbb{Z}_{N}\right]\right|}{N}+\log \left(2 \cos \left((g-1) \theta_{M_{3}, N}\right)\right)\right\}+\ldots
\end{aligned}
$$

Here the dots in the second line represent the contribution from all irreducible flat connections other than geom and geom. We assume that the contribution of non-geometric flat connections to $S_{1}$ is exponentially smaller than that of the geometric connections. ${ }^{8}$ The dots in the last line thus stand for exponentially smaller terms. The angle $\theta_{M_{3}, N}:=2 \operatorname{Im}\left[S_{1}^{\text {geom }}\right]$ represents the phase factor from summing over the Bethe vacua $\alpha=$ geom and $\alpha=\overline{\text { geom}}$. For $M_{3}$ with vanishing $H_{1}\left(M_{3}, \mathbb{Z}_{N}\right)$, the large $N$ analysis was already done in [33] and it was checked that the leading $\mathcal{O}\left(N^{3}\right)$ term nicely matches the entropy of magnetically-charged universal black holes in the holographic dual $\mathrm{AdS}_{4}$. The logarithmic correction is

$$
-(g-1) \log \frac{\left|\operatorname{Hom}\left[\pi_{1}\left(M_{3}\right) \rightarrow \mathbb{Z}_{N}\right]\right|}{N} \stackrel{N \rightarrow \infty}{\longrightarrow}(g-1)\left(1-b_{1}\left(M_{3}\right)\right) \log N .
$$

This also matches the supergravity analysis in (5.5).

\subsection{Refined index: no $N^{3}$ behavior}

From (2.29), (3.3) and (3.8) we obtain

$$
\begin{aligned}
& \mathcal{I}_{\text {top }}\left(q ; \mathcal{T}_{N}\left[M_{3}\right]\right)=\frac{\left|\operatorname{Hom}\left[\pi_{1}\left(M_{3}\right) \rightarrow \mathbb{Z}_{N}\right]\right|}{N} \sum_{\left[\mathcal{A}^{\alpha}\right] \in \frac{\chi_{\text {irred }\left(M_{3} ; N\right)}}{\operatorname{Hom}\left[\pi_{1}\left(M_{3}\right) \rightarrow \mathbb{Z}_{N}\right]}} B^{\alpha}(q) B^{\alpha}\left(q^{-1}\right) \\
& =\frac{\left|\operatorname{Hom}\left[\pi_{1}\left(M_{3}\right) \rightarrow \mathbb{Z}_{N}\right]\right|}{N} B^{\text {geom }}(q) B^{\text {geom }}\left(q^{-1}\right)+\text { c.c. }+(\text { other } \alpha \text { 's }) \\
& =\frac{\left|\operatorname{Hom}\left[\pi_{1}\left(M_{3}\right) \rightarrow \mathbb{Z}_{N}\right]\right|}{N} \exp \left(2 \sum_{n=0}^{\infty}(-\omega)^{2 n} S_{2 n+1}^{\text {geom }}\right)+\ldots \\
& =\frac{\left|\operatorname{Hom}\left[\pi_{1}\left(M_{3}\right) \rightarrow \mathbb{Z}_{N}\right]\right|}{N} \exp \left(-\frac{2 N^{3} \operatorname{vol}\left(M_{3}\right)}{3 \pi}+\text { subleading }\right)+\ldots
\end{aligned}
$$

\footnotetext{
${ }^{8} \mathrm{Such}$ an assumption is justified, a posteriori, because the geometric connections correctly reproduce the black hole entropy. We expect the single-center black hole to dominate the total entropy. Similar assumptions that one particular contribution dominates are made in other cases, including [7].
} 
As before, the dots stand for contributions from other $\alpha$ 's than the geometric ones. We see that for the refined index, unlike for the superconformal index, the contributions from $\mathcal{A}^{\text {geom }}$ and $\mathcal{A}^{\overline{g e o m}}$ are exponentially small in the large $N$ limit. This is compatible with the curious observation we made in section 2.4.1 that the refined twisted index seems to actually vanish at finite $N$. This is also compatible with the non-existence of magneticallycharged black holes with $\mathrm{AdS}_{2} \times S^{2}$ horizon in the universal sector, as will be discussed in subsection 4.2 .

\section{M5-branes wrapped on hyperbolic $M_{3}$ and $\mathcal{N}=2$ gauged supergravity}

To motivate the holographic description of the field theories discussed in the previous sections, we start in eleven dimensions where the M5-brane naturally resides. Since the AdS/CFT dictionary identifies the conformal algebra in field theory with the isometries of spacetime on the gravity side, we expect a gravitational solution given by a backreacted AdS solution in which M5-branes are partly wrapped on $M_{3}$.

The study of various solutions representing the supergravity description of M5-branes wrapping hyperbolic 3-manifolds has a distinguished history starting with [66]. Although much of the emphasis has been on the construction of explicit solutions, starting with $\mathrm{AdS}_{4}$ vacuum solutions and expanding into more general black hole solutions, there are fruitful efforts in the direction of consistent truncations [62,67,68]. Our strategy to describe the consistent truncation is to present the $\mathrm{AdS}_{4}$ vacuum solution in the $11 \mathrm{~d}$ context and then to indicate how the setup generalizes to yield a consistent truncation of 11d supergravity to $4 \mathrm{~d}$ minimal $\mathcal{N}=2$ gauged supergravity with just the graviphoton field.

The central intuition for understanding the 11d origin of M5-branes wrapping hyperbolic 3-manifolds is provided by the Pernici-Sezgin solution to $7 \mathrm{~d}$ gauged supergravity originally constructed in [69]: roughly it discusses solutions of the type $\mathrm{AdS}_{4} \times M_{3}$. The original $11 \mathrm{~d}$ point of view was discussed in $[70],{ }^{9}$ while the $7 \mathrm{~d}$ gauged supergravity point of view can be recovered as a consistent reduction. In $11 \mathrm{~d}$ the solution has the form $\mathrm{AdS}_{4} \times Y_{7}$, where $Y_{7}$ is an $S^{4}$ fibration over the hyperbolic 3-manifold $M_{3}$ [70]:

$$
\begin{aligned}
d s_{11}^{2} & =\lambda^{-1}\left(d s^{2}\left(\operatorname{AdS}_{4}\right)+d s_{7}^{2}\right) \\
& =\lambda^{-1} d s^{2}\left(\operatorname{AdS}_{4}\right)+d s_{4}^{2}\left(\mathcal{M}_{\mathrm{SU}(2)}\right)+\hat{w} \otimes \hat{w}+\frac{\lambda^{2}}{16}\left(\frac{d \rho^{2}}{1-\lambda^{3} \rho^{2}}+\rho^{2} d \psi^{2}\right),
\end{aligned}
$$

where $\lambda$ is the warp factor, $\mathcal{M}_{\mathrm{SU}(2)}$ is a $4 \mathrm{~d}$ space with $\mathrm{SU}(2)$ structure, $\hat{w}$ is a one-form, $\rho$ is a coordinate on an interval, and $\psi \in[0,2 \pi]$ is a coordinate on the circle $S^{1}$. The associated Killing vector $\partial / \partial \psi$ is dual to the $\mathrm{U}(1) \mathrm{R}$-symmetry in field theory.

The particular form of the Pernici-Sezgin solution [69] is (see [70]):

$$
d s_{4}^{2}\left(\mathcal{M}_{\mathrm{SU}(2)}\right)+\hat{w} \otimes \hat{w}=f^{2}(\rho) D Y^{a} \otimes D Y^{a}+g^{2}(\rho) e^{a} \otimes e^{a},
$$

\footnotetext{
${ }^{9}$ See also [71] for a more geometric SU(2)-structure-based approach, and [72] for a concise exposition.
} 
where $Y^{a}(a=1,2,3)$ are constrained coordinates on $S^{2}$ satisfying $Y^{a} Y^{a}=1$, whilst $e^{a}$ is a vielbein on the 3 -manifold $M_{3} \cdot{ }^{10}$ The form of the covariant derivative is

$$
D Y^{a}=d Y^{a}+\omega^{a}{ }_{b} Y^{b},
$$

where $\omega^{a b}$ is the spin connection on $M_{3}$. The supersymmetry conditions dictate

$$
\lambda^{3}=\frac{2}{8+\rho^{2}}, \quad f=\frac{\sqrt{1-\lambda^{3} \rho^{2}}}{2 \sqrt{\lambda}}, \quad g=\frac{1}{2 \sqrt{\lambda}}
$$

and the coordinate $\rho$ covers the interval $[0,2 \sqrt{2}]$. The coordinates $Y^{a}, \rho, \psi$ would form a round $S^{4}$ in the undeformed case, but here they form instead a space we denote by $X_{4}$.

A concrete way to realize the hyperbolic manifold $M_{3}$ is to take a quotient of the hyperbolic plane $\mathbb{H}^{3}$ by a discrete subgroup $\Gamma \in \operatorname{PSL}(2, \mathbb{C})$, namely $M_{3}=\mathbb{H}^{3} / \Gamma$. The background is then

$$
\begin{aligned}
d s_{11}^{2} & =\frac{1}{4 \lambda}\left[4 d s_{\mathrm{AdS}_{4}}^{2}+\frac{d x^{2}+d y^{2}+d z^{2}}{z^{2}}+\frac{8-\rho^{2}}{8+\rho^{2}}\left(D Y^{a}\right)^{2}+\frac{1}{2}\left(\frac{d \rho^{2}}{8-\rho^{2}}+\frac{\rho^{2}}{8+\rho^{2}} d \psi^{2}\right)\right] \\
G_{4} & =\frac{1}{4} d \psi \wedge d\left(\lambda^{-1 / 2} \sqrt{1-\lambda^{3} \rho^{2}} J_{3}\right) .
\end{aligned}
$$

A slightly more general class of solutions involves the standard two-forms $\left(J_{1}, J_{2}, J_{3}\right)$ defining the $\mathrm{SU}(2)$ structure. The ansatz for this class of solutions is [70]

$$
\begin{aligned}
\hat{w} & =g Y^{a} e^{a}, \\
J_{1} & =f g D Y^{a} \wedge e^{a}, \\
J_{2} & =f g \epsilon^{a b c} Y^{a} D Y^{b} \wedge e^{c}, \\
J_{3} & =\frac{1}{2} \epsilon^{a b c} Y^{a}\left(f^{2} D Y^{b} \wedge D Y^{c}-g^{2} e^{b} \wedge e^{c}\right) .
\end{aligned}
$$

To understand the quantization condition of the flux and the identification with the number of M5-branes, we need to consider the four-form field $G_{4}$ of 11d supergravity when restricted to the internal space $X_{4}$. In fact, the $G_{4}$ flux in (4.5) is entirely aligned along $X_{4}$. The free energy can be easily computed [71] to yield ${ }^{11}$

$$
\mathcal{F}=\frac{4 \pi^{3} \int_{Y_{7}} \lambda^{-9 / 2} d \operatorname{vol}_{7}}{\left(2 \pi l_{P}\right)^{9}}=\frac{\pi^{5} \operatorname{vol}\left(M_{3}\right)}{3\left(2 \pi l_{P}\right)^{9}} .
$$

The 4-form flux quantization leads to a relation between Planck's constant $l_{P}$ and $N$ :

$$
N=\frac{1}{\left(2 \pi l_{P}\right)^{3}} \int_{X_{4}} G_{4}=\frac{1}{8 \pi l_{P}^{3}} .
$$

Substituting, we can write the free energy in terms of field theory parameters:

$$
\mathcal{F}=\frac{N^{3}}{3 \pi} \operatorname{vol}\left(M_{3}\right) \text {. }
$$

\footnotetext{
${ }^{10}$ We follow our convention and normalize the metric on $M_{3}$ in such a way that the Ricci scalar is $R=-6$.

${ }^{11}$ We correct a typo in eq. (2.9) of [72].
} 
This result was generalized to account for the free energy corresponding to a squashed 3 -sphere $S_{b}^{3}$ in $[73,74]$ :

$$
\mathcal{F}_{b}=\frac{1}{4}\left(b+\frac{1}{b}\right)^{2} \mathcal{F}
$$

The steps to obtain the $11 \mathrm{~d}$ embedding of $4 \mathrm{~d}$ minimal $\mathcal{N}=2$ gauged supergravity are intuitively clear [67]. ${ }^{12}$ Having identified the Killing vector $\partial / \partial \psi$ as the dual to the $\mathrm{U}(1)$ R-symmetry, we simply shift $d \psi \rightarrow d \psi-A$ in the metric given in equation (4.5), where $A$ is the $4 \mathrm{~d}$ one-form gauge field. The general ansatz for $G_{4}$ is somehow more involved, containing both the field strength $F=d A$ as well as its Hodge dual; it can be found in [67]. For our purpose the most important property is that this generalization does not affect the quantization condition above.

The Einstein-graviphoton part of the action is simply

$$
I=\frac{1}{16 \pi G_{(4)}} \int d^{4} x \sqrt{-g}\left(R+\frac{6}{L^{2}}-\frac{L^{2}}{4} F^{2}\right),
$$

where $G_{(4)}$ is the $4 \mathrm{~d}$ Newton constant — not to be confused with $G_{4}$. Note that in the expression above we have explicitly restored the radius of the AdS vacuum solution, which we had set to one in previous expressions. The theory given by (4.11) is precisely the universal sector discussed recently in [12] in the context of microscopic counting of $\mathrm{AdS}_{4}$ black hole entropy. Here the crucial property is the embedding into M-theory and, more precisely, the scaling of Newton's constant with the number of branes $N$. In the action above, $F$ is the field strength for the $\mathrm{U}(1)$ gauge field in $\mathrm{AdS}_{4}$ that couples to the $\mathrm{U}(1)$ R-symmetry of the boundary CFT. The $4 \mathrm{~d}$ Newton constant $G_{(4)}$ after the consistent truncation is related to $N$ in the following way [62]:

$$
\frac{G_{(4)}}{L^{2}}=\frac{3 \pi^{2}}{2 N^{3} \operatorname{vol}\left(M_{3}\right)} .
$$

This is compatible with the general result $\mathcal{F}=\pi L^{2} / 2 G_{(4)}[71]$.

\subsection{Rotating electrically-charged $\mathrm{AdS}_{4}$ black hole}

The original placement of the rotating solutions in the context of gauged $\mathcal{N}=2$ supergravity was presented in $[78,79]$. In this manuscript we follow the notation of [80] in which the background takes the following form:

$$
\begin{array}{rlrl}
d s^{2} & =-\frac{\Delta_{r}}{W}\left(d t-\frac{a}{\Xi} \sin ^{2} \theta d \phi\right)^{2}+W\left(\frac{d r^{2}}{\Delta_{r}}+\frac{d \theta^{2}}{\Delta_{\theta}}\right)+\frac{\Delta_{\theta} \sin ^{2} \theta}{W}\left(a d t-\frac{\rho^{2}+a^{2}}{\Xi} d \phi\right)^{2} \\
\rho & =r+2 m \sinh ^{2} \delta & \Delta_{\theta} & =1-a^{2} g^{2} \cos ^{2} \theta \\
\Delta_{r} & =r^{2}+a^{2}-2 m r+g^{2} \rho^{2}\left(\rho^{2}+a^{2}\right) & \Xi & =1-a^{2} g^{2} \\
W & =\rho^{2}+a^{2} \cos ^{2} \theta & & \\
A & =\frac{2 m \sinh 2 \delta}{W} \rho\left(d t-\frac{a}{\Xi} \sin ^{2} \theta d \phi\right) . &
\end{array}
$$

\footnotetext{
${ }^{12}$ See also [75-77] for other consistent truncations to minimal $\mathcal{N}=2$ gauged supergravity.
} 
The parameter $g$ simply characterizes the strength of the coupling in gauged supergravity and is inversely proportional to the radius of AdS, $g=L^{-1}$. The physics of this solution is parametrized by $(m, a, \delta)$ which roughly characterize the energy, the angular momentum and the non-extremality or charge of the solution. More precisely: ${ }^{13}$

$$
E=\frac{m}{G_{(4)} \Xi^{2}} \cosh 2 \delta, \quad J=\frac{m a}{G_{(4)} \Xi^{2}} \cosh 2 \delta, \quad Q=\frac{m}{4 G_{(4)} \Xi} \sinh 2 \delta .
$$

This solution has been, of course, generalized to include its embedding in $4 \mathrm{~d} \mathcal{N}=8$ gauged supergravity with gauge group $\mathrm{U}(1)^{4}$. This fact makes it a prime object in the study of the AdS/CFT correspondence due to its relation with ABJM theory [81]. When the four charges with respect to $\mathrm{U}(1)^{4}$ are set equal pairwise, one obtains a solution which is naturally embedded into $4 \mathrm{~d} \mathcal{N}=4$ gauged supergravity with gauge group $\mathrm{U}(1) \times \mathrm{U}(1)$. This is the solution that has been widely discussed in [80], focusing on the supersymmetric limit of the non-extremal solution originally presented in [82]. Technically, in this paper we are concerned with the limit of the solution discussed in [80] when $\delta_{1}=\delta_{2}$ (in their notation) which we simply denote by $\delta$. Versions of these solutions have recently been reviewed in $[83,84]$ with emphasis on the entropy function.

Having a potential microscopic description of these solutions, we are naturally interested in the Bekenstein-Hawking entropy $S_{\mathrm{BH}}$ in the BPS limit where one verifies that $[83,84]$

$$
S_{\mathrm{BH}}=\frac{\pi L}{4 G_{(4)}} \frac{J_{\mathrm{BPS}}}{Q_{\mathrm{BPS}}} .
$$

Due to supersymmetry and extremality, $J_{\mathrm{BPS}}$ and $Q_{\mathrm{BPS}}$ are non-linearly related. The entropy can then more explicitly be written as:

$$
S_{\mathrm{BH}}=\frac{\pi L^{2}}{2 G_{(4)}}\left(\sqrt{1+64 \frac{G_{(4)}^{2}}{L^{2}} Q_{\mathrm{BPS}}^{2}}-1\right) .
$$

The gravitational entropy function $\mathcal{S}$ is the "grand canonical partition function" that reproduces the Bekenstein-Hawking entropy upon Legendre transform:

$$
S_{\mathrm{BH}}\left(Q_{\mathrm{BPS}}\right)=\mathcal{S}(\omega)+\omega J_{\mathrm{BPS}}+(\omega+2 \pi i) 2 L Q_{\mathrm{BPS}}
$$

where $\omega\left(Q_{\mathrm{BPS}}\right)$ is fixed by requiring that $\partial$ (r.h.s. $) / \partial \omega=0$. The entropy function $\mathcal{S}$ also reproduces the non-linear constraint between $J_{\mathrm{BPS}}$ and $Q_{\mathrm{BPS}}$, as the condition that $S_{\mathrm{BH}}$ be real positive $[8,16]$. The entropy function can also be computed from the on-shell action of the solution. In any case, it takes the following form [83, 84]:

$$
\mathcal{S}=i \frac{L^{2}}{8 G_{(4)}} \frac{(\omega+2 \pi i)^{2}}{\omega}=\frac{i N^{3}}{12 \pi^{2}} \operatorname{vol}\left(M_{3}\right) \frac{(\omega+2 \pi i)^{2}}{\omega},
$$

where we used the explicit form of the four-dimensional Newton constant $G_{(4)}$. This expression perfectly matches the field theory computation (3.11). This justifies the use of the field theory description as a microscopic explanation for the entropy of the rotating electrically-charged asymptotically $\mathrm{AdS}_{4}$ black holes described above.

\footnotetext{
${ }^{13}$ In [80] Newton's constant $G_{(4)}$ was set to 1 .
} 


\subsection{Rotating magnetically-charged $\mathrm{AdS}_{4}$ black holes}

There have been many studies regarding rotating magnetically-charged black holes that are the natural candidates for the refined topologically twisted index. In particular, there is one recent publication that discusses these issues in detail and contains a comprehensive list of references [85]. A common theme, since the very beginning of the explorations of rotating magnetically-charged black holes, is that within the confines of the universal sector described by the action (4.11), spherically symmetric black holes do not exist [79]. Indeed, the need to couple to matter was recognized very early on [86].

The absence of such supergravity solutions is compatible with the result on the field theory side that the refined topologically twisted index vanishes. It would be interesting to explored the refined topologically twisted index for the hyperbolic two-plane, in which case there are supergravity solutions that one might hope to match.

\section{Logarithmic corrections from one-loop supergravity}

Logarithmic corrections to the extremal black hole entropy can be computed purely in terms of the low energy data, that is, from the spectrum of massless fields in a given gravitational background. These IR corrections are expected to be reproduced by any candidate to a UV complete description of the gravitational theory. Such an IR window into UV physics has been exploited by Ashoke Sen and collaborators in the context of asymptotically flat black holes whose microscopic description lies in string theory [87, 88]. For asymptotically AdS black holes the microscopic UV complete theory is provided by the dual boundary field theory. It is, therefore, natural to study if the terms logarithmic in $N$ coming from the field theory partition functions are reproduced by one-loop supergravity around the black hole backgrounds. There have been some developments in matching the gravity computation to the coefficient of the $\log N$ term on the field theory side [33, 89-95]. In this section we compute the one-loop logarithmic corrections from the gravity side and confront them with the field-theoretic (UV) results. One of the advantages of working in the context of the $3 \mathrm{~d}-3 \mathrm{~d}$ correspondence is that the logarithmic term is derived analytically, without the recourse to numerical methods as was instead the case in [89, 93, 94].

Let us start by recalling a number of important facts regarding one-loop effective actions on supergravity backgrounds. Our setup is $11 \mathrm{~d}$ supergravity, in which we have an explicit embedding of the solutions describing rotating black holes. We highlight that in odd dimensional spaces only zero-modes and boundary terms can contribute to logarithmic terms. We make the assumption that the whole contribution to the action comes from the asymptotic $\mathrm{AdS}_{4}$ region, as it was the case in [96] for the pure $\mathrm{AdS}_{4}$ solution and in [91] for the magnetically-charged asymptotically $\mathrm{AdS}_{4}$ black hole solution. More directly related to our description of M5-branes wrapping hyperbolic 3-manifolds is the recent discussion in [33].

On very general grounds of diffeomorphic invariance, it can be argued that in odddimensional spacetimes the top Seeley-De Witt coefficient $a_{d / 2}$ vanishes [97]. Therefore, the only contribution to the heat kernel comes from zero-modes. Applied to our case, the one-loop contribution in 11d supergravity comes from the analysis of zero-modes. Similar 
properties have, in fact, been already exploited in the context of the logarithmic corrections to BMPV black holes in [88], where logarithmic contributions can be understood through an effective 5d theory. Similarly, the authors of [96] successfully matched the logarithmic term in the large $N$ expansion of the ABJM free energy on $S^{3}$ with a gravity computation performed in 11d supergravity, essentially reducing to the contribution of a two-form zeromode. Along these lines other matches for magnetically-charged asymptotically $\mathrm{AdS}_{4}$ black holes, dual to the topologically twisted index, were performed in [33, 91].

We will not reproduce all the details of the computation here: the interested reader is referred to $[33,91,96]$ for details. Instead, we will briefly sketch the derivation of the one-loop effective action. Given that the only zero-mode on $\mathrm{AdS}_{4}$ is a 2-form, and assuming that the solution is roughly of the form of a warped product $\mathrm{AdS}_{4} \times M_{3} \times \widetilde{S}^{4}$, we need to decompose the kinetic operator along those three subspaces. In order for the 2-form zeromode on $\mathrm{AdS}_{4}$ to survive, we need to have the corresponding part of the kinetic Laplace-like operator also vanishing. The number of zero-modes then depends on the topology of the full space.

When integrating over zero-modes, there is a factor of $L^{ \pm \beta_{A}}$ for each zero-mode, where $\beta_{A}$ is a coefficient to be determined. The total contribution to the partition function from the zero-modes is then

$$
L^{ \pm \beta_{A} n_{A}^{0}}
$$

where $n_{A}^{0}$ is the total number of zero-modes of the kinetic operator $A$, and the sign depends on whether the operator is fermionic or bosonic. Typically, zero-modes are associated with certain asymptotic symmetries - for example, with gauge transformations that do not vanish at infinity. The key idea in determining $\beta_{A}$ is to find the right integration variables and to count the number of powers of $L$ that such integration measure contributes, when one starts from fields that would naturally be present in the action.

Therefore, in our case, the computation of the one-loop effective action reduces to the computation of the zero-mode scaling $\beta_{A}$ and the total number of zero-modes $n_{A}^{0}$. The most important ingredient in formulating the answer is the number of 2 -form zero modes. A simple way to determine their number is by computing the Euler characteristic of the black hole. In $[33,91]$ it was established that $n_{2}^{0}=2(1-g)$ for a black hole whose horizon is a genus $g$ Riemann surface. It is worth pausing over this result. Note that this number is computed using the non-extremal branch of the solution. Moreover, it is independent of the black hole charges. Therefore, be it for the magnetically-charged or the electrically-charged black holes, we obtain the same result.

The last step before writing down the answer is to recall that to quantize elevendimensional supergravity we need to discuss the quantization of the 3 -form field, $C_{3}$. The action for quantizing a $p$-form $A_{p}$ requires a set of $(p-j)$-form ghost fields, with $j=1, \ldots, p$, and the ghost is Grassmann even (odd) if $j$ is even (odd) [98, 99]. For the $(p-j$ )-form, the Laplacian operator $\left(\Delta_{p-j}\right)^{j+1}$ in the computation of the heat kernel leads to an extra scaling power $-(j+1)$, but otherwise the integration over the zero-modes is unchanged. The result, as in eq. (3.4) of [96], is

$$
\Delta F_{\mathrm{Ghost}}=\sum_{j=0}^{p}(-1)^{j}\left(\beta_{A_{p-j}}-j-1\right) n_{A_{p-j}}^{0} \log L .
$$


The scaling exponent for $p$-forms is easily computed [96], yielding $\beta_{p}=(d-2 p) / 2$ in terms of the total dimension $d$ of spacetime.

The zero-mode contribution in our solution can only come from a ghost 2-form $A_{p-j=2}$ which corresponds to $p=3$ and $j=1$ and it leads to the following one-loop contribution

$$
(-1)^{j}\left(\beta_{2}-j-1\right) n_{2}^{0} \log L=\left(2-\beta_{2}\right) n_{2}^{0} \log L .
$$

The full contribution to the logarithmic terms of the one-loop effective action is thus given only by the 2 -form zero-modes and we have:

$$
\log Z_{1-\text { loop }}=\left(2-\beta_{2}\right) n_{2}^{0} \log L=(2-7 / 2) 2(1-g) \log L=(g-1) \log N,
$$

where, according to the structure of the M5-brane solution, we have $L^{3} \sim N$. When restricting to spherically symmetric horizons $(g=0)$ we find perfect agreement with the field theory result.

In [33] the case when $M_{3}$ admits harmonic one-forms was further discussed. In this case there are extra contributions coming from the supergravity 3 -form potential, because one can construct a zero-mode of $C_{3}$ by combining a 2-form zero-mode on $\mathrm{AdS}_{4}$ with a harmonic 1-form on $M_{3}$. The contribution is as in (5.3), but with $p=3, j=0, \beta_{3}=5 / 2$ and the total number of zero-modes $n_{3}^{0}=2 b_{1}(1-g)$ where $b_{1}$ is the first Betti number of $M_{3}$. Thus, the total contribution to the one-loop partition function takes the form: ${ }^{14}$

$$
\log Z_{1-\operatorname{loop}}=(g-1)\left(1-b_{1}\right) \log N
$$

This results perfectly agrees with the field theory computation of the coefficient of the term logarithmic in $N$. It thus provides strong evidence that the superconformal index captures the black hole entropy beyond leading order.

\section{Conclusions}

In this manuscript we have presented an explicit recipe for, and the computation of, the superconformal index of $\mathcal{N}=2$ supersymmetric field theories denoted by $\mathcal{T}_{N}\left[M_{3}\right]$. Our recipe identifies the superconformal index with certain pertubative invariants of $\operatorname{SL}(N, \mathbb{C})$ Chern-Simons theory via the $3 \mathrm{~d}-3 \mathrm{~d}$ correspondence. One advantage of this setup is that it provides analytical results for the leading term and even for the term logarithmic in $N$. Recent approaches to the microscopic counting of states of rotating electrically-charged asymptotically $\mathrm{AdS}_{4}$ black holes have relied on reducing the field-theoretic object to a matrix model, where the leading order can be easily found but the subleading in $N$ corrections seem like a daunting task $[25,26]$. We showed that the leading contribution to the superconformal index perfectly matches the entropy of rotating electrically-charged black holes in the universal sector of $\mathcal{N}=2$ gauge supergravity. More importantly, we demonstrated that the terms logarithmic in $N$ also match on both sides of the AdS/CFT correspondence.

\footnotetext{
${ }^{14}$ This expression corrects a sign error in [33].
} 
We have also studied the refined topologically twisted index and found agreement between its vanishing on the field theory side and the absence of dual rotating magneticallycharged black holes in the universal sector of $\mathcal{N}=2$ gauged supergravity. Our approach provides strong evidence in favour of the refined index vanishing also for finite $N$. This observation has powerful mathematical implications, and it would be quite interesting to pursue this tantalizing relation between number-theoretic objects and black hole physics.

Our subleading result is a powerful tool that could be applied to other situations where various observables are vying for the genuine description of black hole microstates. The situation with various, a priori, different observables yielding the same leading order expression for the black hole entropy in $\mathrm{AdS}_{4}$ is quite similar to the situation in $\mathrm{AdS}_{5}$. In the context of rotating electrically-charged asymptotically $\mathrm{AdS}_{5}$ black holes there are various observables that, at leading order, yield the same entropy function and, therefore, compete for the microscopic description of the entropy. These observables include: a localization computation on complex backgrounds [16], the superconformal index computation via Bethe Ansatz [18, 100] and a free-field theory approach [17]. It is quite plausible that these observables are equivalent; it is also possible that a subleading in $N$ study has the chance to break this degeneracy. It would be quite appropriate to study this problem in more detail.

\subsection{Future directions}

Let us mention a few open questions that would be nice to address.

Curious observation $\mathcal{I}_{\text {top }}\left(\boldsymbol{q} ; \mathcal{T}_{\boldsymbol{N}}\left[\boldsymbol{M}_{\mathbf{3}}\right]\right)=\mathbf{0}$. We checked that $\mathcal{I}_{\text {top }}\left(q ; \mathcal{T}_{N}\left[M_{3}\right]\right)=0$ to some high power in $\hbar$, for some examples of closed hyperbolic 3-manifold $M_{3}$. Is this just a coincidence? It would be interesting to check if the vanishing holds for arbitrary closed hyperbolic 3-manifolds. A rigorous mathematical proof of the vanishing for infinitely many closed hyperbolic 3 -manifolds at $q=1$ will be reported in [29]. If the answer is positive, we need to understand such a non-trivial property either from $\operatorname{SL}(N, \mathbb{C})$ Chern-Simons theory or from the physics of wrapped M5-branes. In this paper we have offered a physical argument from the viewpoint of wrapped M5-branes.

Going beyond the universal sector in the $3 \mathrm{~d}-3 \mathrm{~d}$ correspondence. In this paper we only considered the $3 \mathrm{~d}-3 \mathrm{~d}$ correspondence for closed 3-manifolds $M_{3}$ without any codimension-two defect. The resulting $3 \mathrm{~d} \mathcal{N}=2$ theory (at sufficiently large $N$ ) only has $\mathrm{SO}(2) \mathrm{R}$-symmetry without any flavor symmetry. Holographic duals for these theories are well-established [66, 69], and allowed us to perform one-loop computations on the gravity side. The system, however, could be generalized by introducing codimension-two defects in the $3 \mathrm{~d}-3 \mathrm{~d}$ setup (2.2). Indeed, the $6 \mathrm{~d} A_{N-1} \mathcal{N}=(2,0)$ theory has regular supersymmetric codimension-two defects. Putting those defects along (3d space-time) $\times$ (a knot $\mathcal{K}$ inside $M_{3}$ ), we can engineer more general classes of $3 \mathrm{~d}$ theories labelled by $\left(M_{3}, \mathcal{K}\right)$ and the types of the regular defects. We have concrete field theoretic constructions of the $3 \mathrm{~d}$ theory only when the codimension-two defect is of "maximal type" $[27,36]$. In order to extend our work and to understand more general classes of supersymmetric black 
holes in $\mathrm{AdS}_{4}$, we need a better understanding on the codimension-two defects in the context of the $3 \mathrm{~d}-3 \mathrm{~d}$ correspondence, both from the gauge theory side and the supergravity side. It would be quite interested to pursue this direction, elaborating on the progress reported in $[72,101]$.

Other geometries. From the point of view of the $3 \mathrm{~d} \mathcal{N}=2$ theories $\mathcal{T}_{N}\left[M_{3}\right]$, there are other types of supersymmetric partition functions one could compute, for instance the lens space index [102] or the partition function on Seifert manifolds [103]. In all those cases, suitable 3d-3d relations could be used to relate supersymmetric partition functions to (possibly Euclidean) supergravity backgrounds.

\section{Acknowledgments}

We are thankful to Sergio Cecotti, Francesca Ferrari, Sergei Gukov, Saebyeok Jeong, Heeyeon Kim, Jun Nian, Pavel Putrov, Chiara Toldo and Kazuya Yonekura for helpful discussions. This work was partially done while one of the authors (DG) was visiting APCTP, Pohang for the workshop "Strings, Branes and Gauge Theories", 17-27 July 2019. We thank APCTP for hospitality. FB is supported in part by the MIUR-SIR grant RBSI1471GJ "Quantum Field Theories at Strong Coupling: Exact Computations and Applications". The research of DG is supported in part by the National Research Foundation of Korea Grant NRF grant 2019R1A2C2004880. DG also acknowledges support by the appointment to the JRG program at the APCTP through the Science and Technology Promotion Fund and Lottery Fund of the Korean Government, as well as support by the Korean Local Governments, Gyeongsangbuk-do Province, and Pohang City. The work of LAPZ is supported in part by the U.S. Department of Energy under grant DE-SC0007859.

\section{A Perturbative invariants $S_{n}^{\alpha}\left[M_{3} ; N=2\right]$ for some $M_{3}$ 's}

Dehn-surgery representation. Every orientable closed 3-manifold can be obtained by Dehn surgery along a link $L$ inside the three-sphere $S^{3}$. The 3-manifold $M_{3}$ obtained by a Dehn surgery along a link $L$ with slopes $\left\{P_{i} / Q_{i}\right\}_{i=1}^{|L|}$ is defined as

$$
\begin{aligned}
M_{3} & =\left(S^{3} \backslash L\right)_{P_{1} / Q_{1}, \ldots, P_{|L|} / Q_{|L|}} \\
& :=\left[\left(S^{3} \backslash L\right) \bigcup(|L| \text { solid-tori })\right] / \sim \text { where the gluing is determined by the slopes } \\
P_{i} \mu_{i} & +Q_{i} \lambda_{i} \in H_{1}\left(\partial\left(S^{3} \backslash L\right), \mathbb{Z}\right) \sim(\text { shrinkable boundary cycle of } i \text {-th solid torus }) .
\end{aligned}
$$

Here $|L|$ is the number of components of the link $L$ and $S^{3} \backslash L$ denotes the link complement obtained from removing a tubular neighborhood of the link $L$ from $S^{3}$. The boundary of the link complement consists of $|L|$ tori. For each boundary torus, there is a canonical choice of basis 1-cycles, called merdian $\mu$ (bounding a disk in the tubular neighborhood of the link) and longitude $\lambda$ (homologous to the link component). In particular, $\mu_{i}$ and $\lambda_{i}$ are the merdian and the longitude of the $i$-th boundary torus, respectively. Then $P_{i}$ and $Q_{i}$ are coprime integers and $P_{i} / Q_{i} \in \mathbb{Q} \cup\{\infty\}$ is called the $i$-th slope of Dehn surgery. 
The procedure of gluing solid tori back to the link complement is called Dehn filling. After Dehn filling, the resulting manifold $M_{3}$ is a closed 3-manifold without any boundary.

Perturbative invariants from state-integral model. Using the topological nature of Chern-Simons theory, its path-integral can be reduced to a finite dimensional integral which is called a state-integral model. See $[50-52,104-107]$ for state-integral models of $\operatorname{SL}(N, \mathbb{C})$ Chern-Simons theory from which the perturbative invariants $\left\{S_{n}^{\alpha}\left[M_{3} ; N\right]\right\}$ can be efficiently computed. The state-integral model turns out to be equal to the localization integral of $\mathcal{T}_{N}\left[M_{3}\right]$ on a squashed 3 -sphere $S_{b}^{3}[108]$ with the identification $\hbar=2 \pi i b^{2}$. Here we give concrete expressions for the state-integral models at $N=2$ corresponding to the three smallest closed hyperbolic 3-manifolds, namely Weeks manifold $=\left(S^{3} \backslash \mathbf{5}_{1}^{2}\right)_{P_{1} / Q_{1}=-5, P_{2} / Q_{2}=-5 / 2}$, $\left(S^{3} \backslash \mathbf{4}_{1}\right)_{P / Q=5}$ and $\left(S^{3} \backslash \mathbf{5}_{1}^{2}\right)_{P_{1} / Q_{1}=-6, P_{2} / Q_{2}=-3 / 2}$. Here $\boldsymbol{4}_{1}$ represents the figure-eight knot while $\mathbf{5}_{1}^{2}$ represents the Whitehead link.

The state-integral is schematically written as

$$
\mathcal{Z}_{\hbar}^{\mathrm{SI}}\left(M_{3} ; N\right)=\int \frac{d^{s} \vec{Z}}{(2 \pi \hbar)^{s / 2}} \mathcal{I}_{\hbar}\left(\vec{Z} ; M_{3}, N\right)
$$

and the integrand can be asymptotically expanded as

$$
\log \mathcal{I}_{\hbar}\left(\vec{Z} ; M_{3}, N\right) \quad \stackrel{\hbar \rightarrow 0}{\longrightarrow} \quad \sum_{n=0}^{\infty} \mathcal{W}_{n}\left(\vec{Z}, \vec{\ell}_{z} ; M_{3}, N\right)
$$

Here $\vec{\ell}_{z} \in \mathbb{Z}^{s}$ comes from the branch cut structure of $\log \mathcal{I}_{\hbar}$ in the asymptotic limit. In particular $\mathcal{W}_{0}$ and $\mathcal{W}_{1}$ depend on the choice of sheet in the following way:

$$
\begin{aligned}
& \mathcal{W}_{0}\left(\vec{\ell}_{z}\right)=\mathcal{W}_{0}\left(\vec{\ell}_{z}=\overrightarrow{0}\right)+2 \pi i \overrightarrow{\ell_{z}} \cdot \vec{Z}, \\
& \mathcal{W}_{1}\left(\vec{\ell}_{z}\right)=\mathcal{W}_{1}\left(\vec{\ell}_{z}=\overrightarrow{0}\right)-2 \pi i \sum_{i=1}^{2}\left(\overrightarrow{\ell_{z}}\right)_{i},
\end{aligned}
$$

and $\mathcal{W}_{n \geq 2}$ are independent of the choice. Integration of the state-integral model along a proper cycle gives the squashed three-sphere partition function of $\mathcal{T}_{N}\left[M_{3}\right]$ theory. The classical part $\mathcal{W}_{0}$ is equal to the twisited superpotential, and the Bethe-vacua of $\mathcal{T}_{N}\left[M_{3}\right]$ are obtained by extremizing it:

$$
\mathcal{S}_{\mathrm{BE}}\left(\mathcal{T}_{N}\left[M_{3}\right]\right)=\left\{\vec{z}\left|\exp \left(\partial_{Z_{i}} \mathcal{W}_{0}\left(\vec{Z}, \overrightarrow{\ell_{z}} ; M_{3}, N\right)\right)\right|_{Z_{i}=\log z_{i}}=1, \text { for } i=1, \ldots, s\right\} .
$$

For each $\vec{z}^{\alpha} \in \mathcal{S}_{\mathrm{BE}}\left(\mathcal{T}_{N}\left[M_{3}\right]\right)$, there is a corresponding saddle point $\vec{Z}^{\alpha}$

$$
\partial_{Z_{i}} \mathcal{W}_{0}\left(\vec{Z}, \vec{\ell}_{z} ; M_{3}, N\right)=0, \quad i=1, \ldots s
$$

for a proper choice of $\vec{\ell}_{z} \in \mathbb{Z}^{s}$. Using the $3 \mathrm{~d}$-3d relation (2.34), each saddle point can be identified with an $\operatorname{SL}(N, \mathbb{C})$ flat connection (modulo tensoring with $\mathbb{Z}_{N}$ flat connections) on $M_{3}$ :

$$
\vec{Z}^{\alpha} \Leftrightarrow\left[\mathcal{A}^{\alpha}\right] \in \frac{\chi_{\text {irred }}\left[N ; M_{3}\right]}{\operatorname{Hom}\left[\pi_{1}\left(M_{3}\right) \rightarrow \operatorname{SL}(N, \mathbb{C})\right]} .
$$


Then, the perturbative invariants $\left\{S_{n}^{\alpha}\left(M_{3} ; N\right)\right\}$ around a flat connection $\mathcal{A}^{\alpha}$ can be obtained from a formal perturbative expansion of the state-integral around the corresponding saddle point $\vec{Z}^{\alpha}$. The precise relation is

$$
\mathcal{Z}_{\hbar}^{\text {SI }}\left(M_{3} ; N\right) \stackrel{\begin{array}{l}
\text { expansion around } \\
\text { saddle point } \vec{Z}^{\alpha}
\end{array}}{\longrightarrow} \sqrt{\frac{\left|\operatorname{Hom}\left[\pi_{1}\left(M_{3}\right) \rightarrow \mathbb{Z}_{N}\right]\right|}{N}} \exp \left(\sum_{n} \hbar^{n-1} S_{n}^{\alpha}\left(M_{3} ; N\right)\right) .
$$

Example: $\boldsymbol{M}_{3}=\left(\boldsymbol{S}^{3} \backslash \mathbf{4}_{1}\right)_{P / Q=5}$. Here $\boldsymbol{4}_{1}$ denotes the figure-eight knot. The corresponding $3 \mathrm{~d}$ gauge theory is given in (2.39) and the state-integral model is given by

$$
\begin{aligned}
\mathcal{Z}_{\hbar}^{\mathrm{SI}}\left(M_{3}=\left(S^{3} \backslash \mathbf{4}_{1}\right)_{5} ; N=2\right) & =\int \frac{d Z}{\sqrt{2 \pi \hbar}} \psi_{\hbar}(Z) \exp \left(-\frac{3}{2 \hbar} Z^{2}+\frac{1}{\hbar}(i \pi+\hbar / 2) Z\right) \\
& =\int \frac{d Z}{\sqrt{2 \pi \hbar}} \mathcal{I}_{\hbar}\left(Z ; M_{3}=\left(S^{3} \backslash \mathbf{4}_{1}\right)_{5}\right) .
\end{aligned}
$$

Here $\psi_{\hbar}(Z)$ is a special functional called quantum dilogarithm (QDL):

$$
\psi_{\hbar}(Z):= \begin{cases}\prod_{r=1}^{\infty} \frac{1-q^{r} e^{-Z}}{1-\tilde{q}^{-r+1} e^{-\tilde{Z}}} & \text { if }|q|<1, \\ \prod_{r=1}^{\infty} \frac{1-\tilde{q}^{r} e^{-\tilde{Z}}}{1-q^{-r+1} e^{-Z}} & \text { if }|q|>1,\end{cases}
$$

with

$$
q:=e^{2 \pi i b^{2}}, \quad \tilde{q}:=e^{2 \pi i b^{-2}}, \quad \tilde{Z}:=\frac{1}{b^{2}} Z .
$$

The asymptotic expansion when $\hbar=2 \pi i b^{2} \rightarrow 0$ is given by

$$
\log \psi_{\hbar}(Z) \stackrel{\hbar \rightarrow 0}{\longrightarrow} \sum_{n=0}^{\infty} \frac{B_{n} \hbar^{n-1}}{n !} \widetilde{\operatorname{Li}}_{2-n}\left(e^{-Z}, \ell_{z}\right) .
$$

Here $B_{n}$ are the Bernoulli numbers with $B_{1}=1 / 2$. Moreover

$$
\begin{aligned}
\widetilde{\operatorname{Li}}_{2}\left(e^{-Z}, \ell_{z}\right) & =\operatorname{Li}_{2}\left(e^{-Z}\right)+2 \pi i \ell_{z} Z \\
\widetilde{\operatorname{Li}}_{1}\left(e^{-Z}, \ell_{z}\right) & =\operatorname{Li}_{1}\left(e^{-Z}\right)-2 \pi i \ell_{z} \\
\widetilde{\operatorname{Li}}_{2-n}\left(e^{-Z}, \ell_{z}\right) & =\operatorname{Li}_{2-n}\left(e^{-Z}\right) \quad(n \geq 2)
\end{aligned}
$$

and $\ell_{z} \in \mathbb{Z}$ is a locally-constant function of $Z$ which comes from the branch cut structure of $\mathrm{Li}_{1,2}$. Using the asymptotic expansion of the QDL, we can asymptotically expand the integrand as follows:

$$
\begin{aligned}
& \mathcal{I}_{\hbar}(Z) \stackrel{\hbar \rightarrow 0}{\longrightarrow} \exp \left(\sum_{n=0}^{\infty} \hbar^{n-1} \mathcal{W}_{n}\left(Z, \ell_{z}\right)\right), \text { where } \\
& \mathcal{W}_{0}\left(Z ; \ell_{z}\right)=\operatorname{Li}_{2}\left(e^{-Z}\right)+i \pi\left(1+2 \ell_{z}\right) Z-\frac{3}{2} Z^{2} \\
& \mathcal{W}_{1}\left(Z ; \ell_{z}\right)=\operatorname{Li}_{1}\left(e^{-Z}\right)-2 \pi i \ell_{z}+\frac{1}{2} Z
\end{aligned}
$$


Then $\mathcal{W}_{0}$ is the twisted superpotential and the Bethe vacua are given by the following algebraic equation in $z:=e^{Z}$ :

$$
\exp \left(\partial_{Z} \mathcal{W}_{0}\right)=1 \quad \Rightarrow \quad \frac{1-z}{z^{4}}=1
$$

For each Bethe vacuum $z^{\alpha}$, there is a corresponding $Z^{\alpha}$ with a proper choice of $\ell_{z}^{\alpha}$ such that

$$
\left.\partial_{Z} \mathcal{W}_{0}\right|_{Z=Z^{\alpha}, \ell_{z}=\ell_{z}^{\alpha}}=0
$$

The Bethe vacua are in one-to-one correspondence with irreducible $\operatorname{SL}(2, \mathbb{C})$ flat connections on $M_{3}$. We can perturbatively expand the state-integral around each saddle point $Z^{\alpha}$ as follows:

$$
\begin{aligned}
& \frac{1}{\sqrt{2}} \exp \left(\sum_{n=0}^{\infty} \hbar^{n-1} S_{n}^{\alpha}\left(M_{3} ; N=2\right)\right)=\int \frac{d(\delta Z)}{\sqrt{2 \pi}} \mathcal{I}_{\hbar}\left(Z^{\alpha}+\hbar^{1 / 2} \delta Z ; M_{3}\right) \\
& =\exp \left(\sum_{n=0}^{\infty} \mathcal{W}_{n}\left(Z^{\alpha}\right)\right) \int \frac{d(\delta Z)}{\sqrt{2 \pi}} \exp \left(-\frac{1}{2} \Pi^{\alpha}(\delta Z)^{2}+\sum_{n=1}^{\infty} \sum_{1 \leq m \leq n+2 ; m-n \in 2 \mathbb{Z}} \hbar^{n / 2} C_{n, m}^{\alpha}(\delta Z)^{m}\right) \\
& =\exp \left(\sum_{n=0}^{\infty} \mathcal{W}_{n}\left(Z^{\alpha}\right)\right) \int \frac{d(\delta Z)}{\sqrt{2 \pi}} \exp \left(-\frac{1}{2} \Pi^{\alpha}(\delta Z)^{2}\right)\left(1+\sum_{n=1}^{\infty} \sum_{m=1}^{3 n} \hbar^{n} D_{n, m}^{\alpha}(\delta Z)^{2 m}\right) \\
& =\exp \left(-\frac{1}{2} \log \Pi^{\alpha}+\sum_{n=0}^{\infty} \mathcal{W}_{n}\left(Z^{\alpha}\right)\right)\left(1+\sum_{n=1}^{\infty} \sum_{m=1}^{3 n} \hbar^{n} D_{n, m}^{\alpha} G_{m}^{\alpha}\right) .
\end{aligned}
$$

Here we defined:

$$
\begin{aligned}
\Pi^{\alpha} & :=-\left.\partial_{Z}^{2} \mathcal{W}_{0}\right|_{Z=Z^{\alpha}} \quad \text { (propagator), } \\
C_{n, m}^{\alpha} & :=\left.\frac{1}{m !} \partial_{Z}^{m} \mathcal{W}_{\frac{n-m}{2}+1}\right|_{Z=Z^{\alpha}} \text { (vertices), } \\
D_{n, m}^{\alpha} & :=\text { coefficient of } \hbar^{n}(\delta Z)^{2 m} \text { in } \exp \left(\sum_{n=1}^{\infty} \sum_{1 \leq m \leq n+2 ; m-n \in 2 \mathbb{Z}} \hbar^{n / 2} C_{n, m}^{\alpha}(\delta Z)^{m}\right), \\
G_{m}^{\alpha} & :=\sqrt{\Pi^{\alpha}} \int \frac{d(\delta Z)}{\sqrt{2 \pi}} \exp \left(-\frac{1}{2} \Pi^{\alpha}(\delta Z)^{2}\right)(\delta Z)^{m}=\left.\partial_{\mu}^{2 m} \exp \left(\frac{1}{2}\left(\Pi^{\alpha}\right)^{-1} \mu^{2}\right)\right|_{\mu=0} .
\end{aligned}
$$

The perturbative coefficients $\left\{S_{n}^{\alpha}\left(M_{3}=\left(S^{3} \backslash \mathbf{4}_{1}\right)_{P / Q=5} ; N=2\right)\right\}$ computed as above are given in (2.40) up to $n=5$.

Example: $\quad M_{3}=$ Weeks manifold $=\left(S^{3} \backslash 5_{1}^{2}\right)_{P_{1} / Q_{1}=-5, P_{2} / Q_{2}=-5 / 2}$. Here $5_{1}^{2}$ denotes the Whitehead link. The corresponding $3 \mathrm{~d}$ gauge theory is given in (2.46). The corresponding state-integral model is given by

$$
\mathcal{Z}_{\hbar}^{\mathrm{SI}}(\mathbf{W e e k s})=\int \frac{d Z}{\sqrt{2 \pi \hbar}} \psi_{\hbar}(Z) \exp \left(-\frac{Z^{2}}{\hbar}\right)
$$




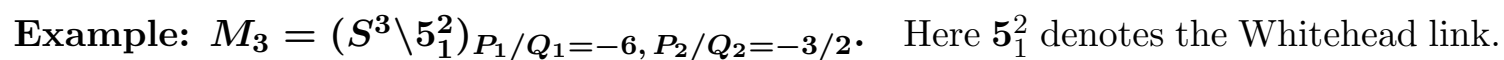
The corresponding $3 \mathrm{~d}$ gauge theory is given in (2.52), and the state-integral model is

$$
\mathcal{Z}_{\hbar}^{\mathrm{SI}}\left(M_{3}=\left(S^{3} \backslash \mathbf{5}_{1}^{2}\right)_{P_{1} / Q_{1}=-6, P_{2} / Q_{2}=-3 / 2}\right)=\int \frac{d Z}{\sqrt{2 \pi \hbar}} \psi_{\hbar}(Z) \exp \left(-\frac{Z^{2}}{2 \hbar}\right) .
$$

Open Access. This article is distributed under the terms of the Creative Commons Attribution License (CC-BY 4.0), which permits any use, distribution and reproduction in any medium, provided the original author(s) and source are credited.

\section{References}

[1] J.D. Bekenstein, Black holes and the second law, Lett. Nuovo Cim. 4 (1972) 737 [InSPIRE].

[2] J.D. Bekenstein, Black holes and entropy, Phys. Rev. D 7 (1973) 2333 [InSPIRE].

[3] J.D. Bekenstein, Generalized second law of thermodynamics in black hole physics, Phys. Rev. D 9 (1974) 3292 [INSPIRE].

[4] S.W. Hawking, Black hole explosions, Nature 248 (1974) 30 [INSPIRE].

[5] S.W. Hawking, Particle Creation by Black Holes, Commun. Math. Phys. 43 (1975) 199 [Erratum ibid. 46 (1976) 206] [INSPIRE].

[6] A. Strominger and C. Vafa, Microscopic origin of the Bekenstein-Hawking entropy, Phys. Lett. B 379 (1996) 99 [hep-th/9601029] [INSPIRE].

[7] F. Benini, K. Hristov and A. Zaffaroni, Black hole microstates in $A d S_{4}$ from supersymmetric localization, JHEP 05 (2016) 054 [arXiv:1511.04085] [INSPIRE].

[8] F. Benini, K. Hristov and A. Zaffaroni, Exact microstate counting for dyonic black holes in AdS4, Phys. Lett. B 771 (2017) 462 [arXiv:1608.07294] [InSPIRE].

[9] F. Benini and A. Zaffaroni, A topologically twisted index for three-dimensional supersymmetric theories, JHEP 07 (2015) 127 [arXiv: 1504.03698] [INSPIRE].

[10] S.M. Hosseini and A. Zaffaroni, Large $N$ matrix models for $3 d \mathcal{N}=2$ theories: twisted index, free energy and black holes, JHEP 08 (2016) 064 [arXiv: 1604.03122] [INSPIRE].

[11] A. Cabo-Bizet, V.I. Giraldo-Rivera and L.A. Pando Zayas, Microstate counting of AdS hyperbolic black hole entropy via the topologically twisted index, JHEP 08 (2017) 023 [arXiv: 1701.07893] [INSPIRE].

[12] F. Azzurli, N. Bobev, P.M. Crichigno, V.S. Min and A. Zaffaroni, A universal counting of black hole microstates in $A d S_{4}, J H E P 02$ (2018) 054 [arXiv:1707.04257] [INSPIRE].

[13] S.M. Hosseini, K. Hristov and A. Passias, Holographic microstate counting for AdS 4 black holes in massive IIA supergravity, JHEP 10 (2017) 190 [arXiv:1707.06884] [INSPIRE].

[14] F. Benini, H. Khachatryan and P. Milan, Black hole entropy in massive Type IIA, Class. Quant. Grav. 35 (2018) 035004 [arXiv:1707.06886] [INSPIRE].

[15] S.M. Hosseini, A. Nedelin and A. Zaffaroni, The Cardy limit of the topologically twisted index and black strings in $A d S_{5}$, JHEP 04 (2017) 014 [arXiv: 1611.09374] [INSPIRE].

[16] A. Cabo-Bizet, D. Cassani, D. Martelli and S. Murthy, Microscopic origin of the Bekenstein-Hawking entropy of supersymmetric AdS $S_{5}$ black holes, JHEP 10 (2019) 062 [arXiv: 1810.11442] [INSPIRE]. 
[17] S. Choi, J. Kim, S. Kim and J. Nahmgoong, Large AdS black holes from QFT, arXiv: 1810.12067 [INSPIRE].

[18] F. Benini and P. Milan, Black holes in $4 d \mathcal{N}=4$ Super-Yang-Mills, arXiv:1812.09613 [INSPIRE].

[19] S.M. Hosseini, I. Yaakov and A. Zaffaroni, Topologically twisted indices in five dimensions and holography, JHEP 11 (2018) 119 [arXiv:1808.06626] [INSPIRE].

[20] P.M. Crichigno, D. Jain and B. Willett, 5d Partition Functions with A Twist, JHEP 11 (2018) 058 [arXiv: 1808.06744] [INSPIRE].

[21] S.M. Hosseini, K. Hristov, A. Passias and A. Zaffaroni, 6D attractors and black hole microstates, arXiv:1809.10685 [INSPIRE].

[22] M. Fluder, S.M. Hosseini and C.F. Uhlemann, Black hole microstate counting in Type IIB from 5d SCFTs, JHEP 05 (2019) 134 [arXiv: 1902.05074] [INSPIRE].

[23] G. Kántor, C. Papageorgakis and P. Richmond, AdS 7 black-hole entropy and $5 D \mathcal{N}=2$ Yang-Mills, JHEP 01 (2020) 017 [arXiv: 1907.02923] [INSPIRE].

[24] A. Zaffaroni, Lectures on AdS Black Holes, Holography and Localization, 2019, arXiv: 1902.07176 [INSPIRE].

[25] S. Choi, C. Hwang and S. Kim, Quantum vortices, M2-branes and black holes, arXiv: 1908.02470 [INSPIRE].

[26] J. Nian and L.A. Pando Zayas, Microscopic Entropy of Rotating Electrically Charged $A d S_{4}$ Black Holes from Field Theory Localization, arXiv:1909.07943 [INSPIRE].

[27] T. Dimofte, D. Gaiotto and S. Gukov, Gauge Theories Labelled by Three-Manifolds, Commun. Math. Phys. 325 (2014) 367 [arXiv:1108.4389] [INSPIRE].

[28] T. Dimofte, D. Gaiotto and S. Gukov, 3-Manifolds and 3d Indices, Adv. Theor. Math. Phys. 17 (2013) 975 [arXiv: 1112.5179] [INSPIRE].

[29] D. Gang, S. Kim and S. Yoon, Adjoint Reidemeister torsions from wrapped M5-branes, arXiv: 1911.10718 [INSPIRE].

[30] N. Bobev and P.M. Crichigno, Universal spinning black holes and theories of class $\mathcal{R}$, JHEP 12 (2019) 054 [arXiv: 1909.05873] [INSPIRE].

[31] S. Lee and M. Yamazaki, 3d Chern-Simons Theory from M5-branes, JHEP 12 (2013) 035 [arXiv: 1305.2429] [INSPIRE].

[32] J. Yagi, 3d TQFT from 6d SCFT, JHEP 08 (2013) 017 [arXiv: 1305.0291] [InSPIRE].

[33] D. Gang, N. Kim and L.A. Pando Zayas, Precision Microstate Counting for the Entropy of Wrapped M5-branes, arXiv:1905.01559 [InSPIRE].

[34] D. Gang, Y. Tachikawa and K. Yonekura, Smallest 3d hyperbolic manifolds via simple $3 d$ theories, Phys. Rev. D 96 (2017) 061701 [arXiv:1706.06292] [INSPIRE].

[35] D. Gang and K. Yonekura, Symmetry enhancement and closing of knots in 3d/3d correspondence, JHEP 07 (2018) 145 [arXiv:1803.04009] [INSPIRE].

[36] T. Dimofte, M. Gabella and A.B. Goncharov, K-Decompositions and 3d Gauge Theories, JHEP 11 (2016) 151 [arXiv:1301.0192] [InSPIRE].

[37] E. Witten, Analytic Continuation Of Chern-Simons Theory, AMS/IP Stud. Adv. Math. 50 (2011) 347 [arXiv: 1001.2933 [INSPIRE]. 
[38] R-torsion and the Laplacian on Riemannian manifolds, Adv. Math. 7 (1971) 145 [INSPIRE].

[39] S. Gukov and H. Murakami, SL $(2, \mathbb{C})$ Chern-Simons theory and the asymptotic behavior of the colored Jones polynomial, Lett. Math. Phys. 86 (2008) 79 [math/0608324] [INSPIRE].

[40] C. Beem, T. Dimofte and S. Pasquetti, Holomorphic Blocks in Three Dimensions, JHEP 12 (2014) 177 [arXiv:1211.1986] [INSPIRE].

[41] D. Gang, E. Koh, S. Lee and J. Park, Superconformal Index and 3d-3d Correspondence for Mapping Cylinder/Torus, JHEP 01 (2014) 063 [arXiv:1305.0937] [INSPIRE].

[42] H.-J. Chung, T. Dimofte, S. Gukov and P. Sułkowski, 3d-3d Correspondence Revisited, JHEP 04 (2016) 140 [arXiv: 1405.3663] [InSPIRE].

[43] T. Dimofte, Perturbative and nonperturbative aspects of complex Chern-Simons theory, J. Phys. A 50 (2017) 443009 [arXiv: 1608. 02961] [INSPIRE].

[44] F. Nieri and S. Pasquetti, Factorisation and holomorphic blocks in 4d, JHEP 11 (2015) 155 [arXiv: 1507.00261] [INSPIRE].

[45] F. Benini and S. Cremonesi, Partition Functions of $\mathcal{N}=(2,2)$ Gauge Theories on $S^{2}$ and Vortices, Commun. Math. Phys. 334 (2015) 1483 [arXiv:1206.2356] [INSPIRE].

[46] F. Benini and W. Peelaers, Higgs branch localization in three dimensions, JHEP 05 (2014) 030 [arXiv: 1312.6078] [INSPIRE].

[47] S. Gukov, D. Pei, P. Putrov and C. Vafa, BPS spectra and 3-manifold invariants, arXiv: 1701.06567 [INSPIRE].

[48] F. Benini and A. Zaffaroni, Supersymmetric partition functions on Riemann surfaces, Proc. Symp. Pure Math. 96 (2017) 13 [arXiv:1605.06120] [INSPIRE].

[49] C. Closset and H. Kim, Comments on twisted indices in 3d supersymmetric gauge theories, JHEP 08 (2016) 059 [arXiv: 1605.06531] [INSPIRE].

[50] T. Dimofte, S. Gukov, J. Lenells and D. Zagier, Exact Results for Perturbative Chern-Simons Theory with Complex Gauge Group, Commun. Num. Theor. Phys. 3 (2009) 363 [arXiv: 0903.2472] [INSPIRE].

[51] T.D. Dimofte and S. Garoufalidis, The quantum content of the gluing equations, Geom. Topol. 17 (2013) 1253 [arXiv:1202.6268] [INSPIRE].

[52] D. Gang, M. Romo and M. Yamazaki, All-Order Volume Conjecture for Closed 3-Manifolds from Complex Chern-Simons Theory, Commun. Math. Phys. 359 (2018) 915 [arXiv: 1704.00918] [INSPIRE].

[53] H.-C. Kim and S. Kim, Supersymmetric vacua of mass-deformed M2-brane theory, Nucl. Phys. B 839 (2010) 96 [arXiv:1001.3153] [INSPIRE].

[54] K. Intriligator and N. Seiberg, Aspects of $3 d N=2$ Chern-Simons-Matter Theories, JHEP 07 (2013) 079 [arXiv: 1305.1633] [INSPIRE].

[55] D. Gaiotto, $N=2$ dualities, JHEP 08 (2012) 034 [arXiv:0904.2715] [INSPIRE].

[56] F. Benini, Y. Tachikawa and D. Xie, Mirrors of 3d Sicilian theories, JHEP 09 (2010) 063 [arXiv: 1007.0992] [INSPIRE].

[57] S. Gukov, P. Putrov and C. Vafa, Fivebranes and 3-manifold homology, JHEP 07 (2017) 071 [arXiv: 1602.05302] [INSPIRE].

[58] D. Gabai, R. Meyerhoff and P. Milley, Minimum volume cusped hyperbolic three-manifolds, J. Am. Math. Soc. 22 (2009) 1157 arXiv:0705. 4325. 
[59] G.D. Mostow, Quasi-conformal mappings in n-space and the rigidity of hyperbolic space forms, Publications Mathématiques de l'IHÉS 34 (1968) 53.

[60] W. Müller, The asymptotics of the Ray-Singer analytic torsion of hyperbolic 3-manifolds, in Metric and differential geometry, Prog. Math. 297 (2012) 317 [arXiv:1003.5168].

[61] J. Park, Reidemeister torsion, complex volume and Zograf infinite product for hyperbolic 3-manifolds with cusps, arXiv:1712.08270.

[62] D. Gang, N. Kim and S. Lee, Holography of 3d-3d correspondence at Large N, JHEP 04 (2015) 091 [arXiv: 1409.6206] [InSPIRE].

[63] T. Dimofte and S. Gukov, Chern-Simons Theory and S-duality, JHEP 05 (2013) 109 [arXiv: 1106.4550$]$ [INSPIRE].

[64] D. Gang and Y. Hatsuda, S-duality resurgence in SL(2) Chern-Simons theory, JHEP 07 (2018) 053 [arXiv: 1710.09994] [INSPIRE].

[65] S.M. Hosseini, Black hole microstates and supersymmetric localization, Ph.D. thesis, Milan Bicocca University, (2018) arXiv:1803.01863 [INSPIRE].

[66] J.P. Gauntlett, N. Kim and D. Waldram, M Five-branes wrapped on supersymmetric cycles, Phys. Rev. D 63 (2001) 126001 [hep-th/0012195] [INSPIRE].

[67] J.P. Gauntlett and O. Varela, Consistent Kaluza-Klein reductions for general supersymmetric AdS solutions, Phys. Rev. D 76 (2007) 126007 [arXiv:0707.2315] [INSPIRE].

[68] A. Donos, J.P. Gauntlett, N. Kim and O. Varela, Wrapped M5-branes, consistent truncations and AdS/CMT, JHEP 12 (2010) 003 [arXiv:1009.3805] [INSPIRE].

[69] M. Pernici and E. Sezgin, Spontaneous Compactification of Seven-dimensional Supergravity Theories, Class. Quant. Grav. 2 (1985) 673 [INSPIRE].

[70] J.P. Gauntlett, O.A.P. Mac Conamhna, T. Mateos and D. Waldram, AdS spacetimes from wrapped M5 branes, JHEP 11 (2006) 053 [hep-th/0605146] [INSPIRE].

[71] M. Gabella, D. Martelli, A. Passias and J. Sparks, $\mathcal{N}=2$ supersymmetric AdS 4 solutions of M-theory, Commun. Math. Phys. 325 (2014) 487 [arXiv:1207.3082] [INSPIRE].

[72] I. Bah, M. Gabella and N. Halmagyi, BPS M5-branes as Defects for the $3 d$-3d Correspondence, JHEP 11 (2014) 112 [arXiv:1407.0403] [INSPIRE].

[73] D. Martelli, A. Passias and J. Sparks, The gravity dual of supersymmetric gauge theories on a squashed three-sphere, Nucl. Phys. B 864 (2012) 840 [arXiv:1110.6400] [INSPIRE].

[74] D. Gang, N. Kim and S. Lee, Holography of wrapped M5-branes and Chern-Simons theory, Phys. Lett. B 733 (2014) 316 [arXiv:1401.3595] [InSPIRE].

[75] G. Larios, P. Ntokos and O. Varela, Embedding the $\mathrm{SU}(3)$ sector of $\mathrm{SO}(8)$ supergravity in $D=11$, Phys. Rev. D 100 (2019) 086021 [arXiv:1907.02087] [InSPIRE].

[76] G. Larios and O. Varela, Minimal $D=4 \mathcal{N}=2$ supergravity from $D=11$ : An M-theory free lunch, JHEP 10 (2019) 251 [arXiv:1907.11027] [INSPIRE].

[77] O. Varela, Minimal D = 4 truncations of type IIA, JHEP 11 (2019) 009 [arXiv: 1908.00535] [InSPIRE].

[78] V.A. Kostelecky and M.J. Perry, Solitonic black holes in gauged $N=2$ supergravity, Phys. Lett. B 371 (1996) 191 [hep-th/9512222] [INSPIRE]. 
[79] M.M. Caldarelli and D. Klemm, Supersymmetry of Anti-de Sitter black holes, Nucl. Phys. B 545 (1999) 434 [hep-th/9808097] [INSPIRE].

[80] M. Cvetič, G.W. Gibbons, H. Lü and C.N. Pope, Rotating black holes in gauged supergravities: Thermodynamics, supersymmetric limits, topological solitons and time machines, hep-th/0504080 [INSPIRE].

[81] O. Aharony, O. Bergman, D.L. Jafferis and J. Maldacena, $N=6$ superconformal Chern-Simons-matter theories, M2-branes and their gravity duals, JHEP 10 (2008) 091 [arXiv:0806.1218] [INSPIRE].

[82] Z.W. Chong, M. Cvetič, H. Lü and C.N. Pope, Charged rotating black holes in four-dimensional gauged and ungauged supergravities, Nucl. Phys. B 717 (2005) 246 [hep-th/0411045] [INSPIRE].

[83] S. Choi, C. Hwang, S. Kim and J. Nahmgoong, Entropy functions of BPS black holes in $A d S_{4}$ and $A d S_{6}$, J. Korean Phys. Soc. 76 (2020) 101 [arXiv: 1811.02158] [INSPIRE].

[84] D. Cassani and L. Papini, The BPS limit of rotating AdS black hole thermodynamics, JHEP 09 (2019) 079 [arXiv: 1906.10148] [INSPIRE].

[85] K. Hristov, S. Katmadas and C. Toldo, Rotating attractors and BPS black holes in $A d S_{4}$, JHEP 01 (2019) 199 [arXiv: 1811.00292] [INSPIRE].

[86] D. Klemm, Rotating BPS black holes in matter-coupled AdS 4 supergravity, JHEP 07 (2011) 019 [arXiv: 1103.4699] [INSPIRE].

[87] A. Sen, Logarithmic Corrections to $N=2$ Black Hole Entropy: An Infrared Window into the Microstates, Gen. Rel. Grav. 44 (2012) 1207 [arXiv:1108.3842] [INSPIRE].

[88] A. Sen, Logarithmic Corrections to Rotating Extremal Black Hole Entropy in Four and Five Dimensions, Gen. Rel. Grav. 44 (2012) 1947 [arXiv:1109.3706] [INSPIRE].

[89] J.T. Liu, L.A. Pando Zayas, V. Rathee and W. Zhao, Toward Microstate Counting Beyond Large $N$ in Localization and the Dual One-loop Quantum Supergravity, JHEP 01 (2018) 026 [arXiv: 1707.04197] [INSPIRE].

[90] I. Jeon and S. Lal, Logarithmic Corrections to Entropy of Magnetically Charged AdS4 Black Holes, Phys. Lett. B 774 (2017) 41 [arXiv:1707.04208] [INSPIRE].

[91] J.T. Liu, L.A. Pando Zayas, V. Rathee and W. Zhao, One-Loop Test of Quantum Black Holes in anti-de Sitter Space, Phys. Rev. Lett. 120 (2018) 221602 [arXiv:1711.01076] [INSPIRE].

[92] K. Hristov, I. Lodato and V. Reys, On the quantum entropy function in $4 d$ gauged supergravity, JHEP 07 (2018) 072 [arXiv: 1803.05920] [INSPIRE].

[93] J.T. Liu, L.A. Pando Zayas and S. Zhou, Subleading Microstate Counting in the Dual to Massive Type IIA, arXiv: 1808.10445 [INSPIRE].

[94] L.A. Pando Zayas and Y. Xin, Topologically twisted index in the 't Hooft limit and the dual AdS $S_{4}$ black hole entropy, Phys. Rev. D 100 (2019) 126019 [arXiv:1908.01194] [INSPIRE].

[95] K. Hristov, I. Lodato and V. Reys, One-loop determinants for black holes in 4 d gauged supergravity, JHEP 11 (2019) 105 [arXiv: 1908.05696] [INSPIRE].

[96] S. Bhattacharyya, A. Grassi, M. Mariño and A. Sen, A One-Loop Test of Quantum Supergravity, Class. Quant. Grav. 31 (2014) 015012 [arXiv:1210.6057] [INSPIRE]. 
[97] D.V. Vassilevich, Heat kernel expansion: User's manual, Phys. Rept. 388 (2003) 279 [hep-th/0306138] [INSPIRE].

[98] W. Siegel, Hidden Ghosts, Phys. Lett. 93B (1980) 170 [InSPIRE].

[99] E.J. Copeland and D.J. Toms, Quantized Antisymmetric Tensor Fields and Selfconsistent Dimensional Reduction in Higher Dimensional Space-times, Nucl. Phys. B 255 (1985) 201 [INSPIRE].

[100] F. Benini and P. Milan, A Bethe Ansatz type formula for the superconformal index, arXiv: 1811.04107 [INSPIRE].

[101] D. Gang, N. Kim, M. Romo and M. Yamazaki, Aspects of Defects in 3d-3d Correspondence, JHEP 10 (2016) 062 [arXiv:1510.05011] [InSPIRE].

[102] F. Benini, T. Nishioka and M. Yamazaki, 4d Index to 3d Index and 2d TQFT, Phys. Rev. D 86 (2012) 065015 [arXiv:1109.0283] [INSPIRE].

[103] C. Closset, H. Kim and B. Willett, Seifert fibering operators in $3 d \mathcal{N}=2$ theories, JHEP 11 (2018) 004 [arXiv: 1807.02328] [INSPIRE].

[104] K. Hikami, Hyberbolic structure arising from a knot invariant, Int. J. Mod. Phys. A 16 (2001) 3309 math-ph/0105039.

[105] K. Hikami, Generalized Volume Conjecture and the A-Polynomials: The Neumann-Zagier Potential Function as a Classical Limit of Quantum Invariant, J. Geom. Phys. 57 (2007) 1895 [math/0604094] [INSPIRE].

[106] T. Dimofte, Quantum Riemann Surfaces in Chern-Simons Theory, Adv. Theor. Math. Phys. 17 (2013) 479 [arXiv:1102.4847] [InSPIRE].

[107] J.-B. Bae, D. Gang and J. Lee, $3 d \mathcal{N}=2$ minimal SCFTs from Wrapped M5-branes, JHEP 08 (2017) 118 [arXiv: 1610.09259] [INSPIRE].

[108] N. Hama, K. Hosomichi and S. Lee, SUSY Gauge Theories on Squashed Three-Spheres, JHEP 05 (2011) 014 [arXiv:1102.4716] [INSPIRE]. 